\title{
Effects of Football Match Results of Croatian National Team on Stock Returns: Evidence from Zagreb Stock Exchange
}

\author{
Tihana Škrinjarić ${ }^{*}$ \\ Patrik Barišic ***
}

\begin{abstract}
This paper observes short term effects of football match results by focusing on the Croatian national team and stock returns on the Zagreb Stock Exchange. Existing literature identifies psychological factors affecting investor's sentiment around sporting events on different stock markets. There does not exist any study focusing on the Croatian stock market. Thus, this paper extensively observes such effects for the first time in the literature. Event study methodology, a usual approach of investigating such effects, is used on a sample of 60 stocks on the Zagreb Stock Exchange for the period from 2014 until the end of 2018. The results indicate no significant effects of winning or losing a football match, even when controlling for game being friendly, competitive tournament or qualification one; as well as after controlling for investor's expectations based upon betting odds. This means that no profitable trading strategies could be obtained around the football match day on the Zagreb Stock Exchange in the observed period for the stocks investigated in this paper.
\end{abstract}

Keywords: investor sentiment; football results; event study; stock reactions; investor's expectations; betting odds

JEL Classification: G12, C10, G14

\section{Introduction}

Observing the effects of football match results on stock markets has obtained great popularity in the recent decade. Literature in this field belongs to the area of behavioural finance theory due to analysing the investor's mood around certain dates and how the sentiment related to the national pride spills over to the decision making process in portfolio management. If effects of football match outcomes are found in

\footnotetext{
* Tihana Škrinjarić is at Faculty of Economics and Business, University of Zagreb, Zagreb, Croatia.

** Patrik Barišić is a student of integrated university study programme at Faculty of Economics and Business, University of Zagreb, Zagreb, Croatia.
} 
stock returns (volatilities, volumes, etc.), then it could be said that the semi strong form of the Efficient Market Hypothesis (Fama 1965, 1970) is violated due to existence of exploitable strategies based upon the stock market reactions around specific dates. Many rational asset pricing models assume rational behaviour of investors. However, there is an enormous body of literature within the field of behavioural finance which shows that at least sometimes, this cannot hold. Indeed, a vast amount of literature exists which shows that peoples' mood significantly affects the decision making process of economic agents (see Johnson and Tversky 1983 or Lowenstein et al. 2001 for introduction). Lowenstein (2000) focused on visceral factors (emotions and feelings) when making economic decisions. Author showed that ignoring such factors in rational economic models leads to misspecification of results and such models can be used in practice only when visceral factors do not affect people. Such analysis dates back to late 18th century (when Bentham 1789 observed individual's utility function as a net sum of positive and negative emotions). Today, many different anomalies in investors' behaviour and stock prices can be found across literature; such as calendar anomalies, lunar cycle effects, seasonal affective disorder as some of the popular ones. Overview of some of the greatest problems within the rational approach of financial modelling can be found in the extensive research of Hirshleifer (2001).

This paper belongs to the growing literature in which it is assumed and tested that different factors such as the weather, emotions, psychological state, etc. have an impact on decision making process of people and investors. Such literature stems from the psychology literature which found significant results of positive emotions, mood and pride of sport fans after a winning match in different sports. Examples include early work of Isen and Simmonds (1978) and Isen, Nyugen and Ashby (1988), in which groups of people were compared with respect to peoples' mood and utility functions in gambling scenarios. Namely, the results indicated that happy people tend to overestimate the probability of winning compared to control groups. This can be reflected around winning football matches results on stock markets. Sporting outcomes and identification with the sport team was studied in Wann et al. (1994); where high-identification people (i.e. fans) exhibited the most intense reactions to outcomes of matches. This was found true for both the winning and losing outcomes. Work of Edmans et al. (2007) popularized the approach to looking at sporting events from the combination of psychological and sentiment point of view. Authors focused on the football results as a mood variable which affects investors' sentiment due to satisfying three characteristics: football results drive the mood substantially and unambiguously; these results affect a large proportion of the population (and thus enough investors) and its effects are correlated across majority of people. As it will be seen in the second section, more and more research has been emerging since 2007 which focuses on the effects of sport outcomes on the investors' mood, i.e. how it reflects in stock returns around crucial dates. Bernile and Lyandres (2011) describe the investor sentiment around sporting outcomes based upon prices of contracts traded on betting 
exchanges. Within this approach, authors found that pre-event prices are inefficient and they become efficient after the event.

The goal of this paper is to provide a comprehensive study of the effects of football match results in Croatia for the first time in the literature. Firstly, this research observes the typical breakdown of results into wins; draws and losses as previous literature observes. Next, this study focus on the results for the competitive and qualification matches and compare them to the friendly ones in order to see if the greater the stake, the greater stock return reactions to the match results. The results are controlled for Monday effects in the analysis, due to previous literature finding it significant on the Croatian market ${ }^{1}$. Robustness of results is checked with several approaches, including the pre-match expectations of investors, by including probability of winning via betting odds. The motivation can be found in the fact that there does not exist such type of study for the Croatia up until writing this research. There are several reasons on why the focus is on Croatia. Croatia is a sporting nation and reason why this paper observes effects of football match results of Croatian national football team on stock returns on the Croatian stock exchange is because of huge level of popularity of football in the country. Football is the most popular sport in Croatia nowadays with around 1.500 registered clubs and 128.000 participants (this is around $3 \%$ of total population in the country). Football manifestations are gathering huge masses and entering into different spheres of human life such as politics, culture and economics. Interested readers are referred to Lalić (2018) on the history on Croatian football and politics. By looking the investors' point of view, although this market is illiquid, previous literature found evidence in favour of it not being efficient in terms of the Efficient Market Hypothesis ${ }^{2}$. In that way, it is interesting to find out if sporting events affect investor's mood and expectations and reflect in stock prices, i.e. returns. Although low liquidity is often mentioned in the literature as being a negative characteristic of markets such as the Croatian one, in their detailed review of Central and Eastern European stock markets Baele, Bekaert and Schäfer (2015) established that there exist illiquidity premiums on such markets. Moreover, this type of research makes sense to do on ZSE. There exists evidence that mispricing is sometimes greater in liquid markets compared to illiquid ones (see Bloomfield, O'Hara, and Saar (2009), Linnainmaa (2007), and Tetlock (2011)). Tetlock (2008) even controls for different security characteristics (volatility, time until expiration, etc.) when looking the relationship between mispricing and liquidity and found that liquidity is not the issue when pricing securities. This research was even done on limit orders regarding the sport prediction, related to studies such as this one. It is explained that illiquidity precedes the release of new information about fundamental values of stocks which means that investors are reluctant to submit limit orders. 
Thus, the rest of the paper is structured as follows. Second section gives an overview of the previous recent related literature. Third section describes the methodology used in the study with the results given in the fourth section. The fifth section discusses the obtained results and the final section concludes the paper.

\section{Previous literature review}

Existing literature on effects of sport events on investors' sentiment today is enormous. It includes different methodologies, countries, sporting events and sports. Since the literature is growing at a rapid pace, this section mentions some of the more recent interesting results, related to this study, namely, football match results on stock returns. Other sporting events have been observed in the literature as well (see Brown and Sauer 1993 for basketball; Zawadziki 2013 for winter Olympic games). However, football is the most researched sport due to it being the most popular sport in general to follow. Some research did not find significant results when observing the effects of sporting outcomes on stock markets, such as Klein, Zwergel and Heiden (2009) where authors found that there is no connection between football match results and stock index returns in Europe. Gallagher and O'Sullivan (2011) also found that there is no link between sport results and stock market returns in case of Ireland. Berkowitz and Depken (2017) confirmed an asymmetric market reaction to winning and losing and that the stock market responds stronger and slower to losing than to winning. Further, Tufan (2004) investigated whether World cup matches have an impact on ISE 100 Index returns. Author found no significant relationship. Boyle and Walter (2003) also found no evidence between sporting team success and stock market return. Other examples of no effects include Zuber et al. (2005) for English clubs and Gerlach (2011) for variety of countries in the study.

Significant effects were found in the following literature. Ashton, Gerrard and Hudson (2003) investigated relationship between the performance of English national team and FTSE-100 stock market returns (period: 1984-2002). Results indicate that good (bad) results by English national team are followed by good (bad) market returns ${ }^{3}$. Scholtens and Peenstra (2009) analyzed the effect of results of national and European football matches on stock market performance of eight different countries (sample: 2000-2004). Firstly, authors concluded that the stock market responds positive to victories and negative to defeats. Secondly, the stock market responds asymmetrically, i.e. the response to defeat is stronger than that to victory. Unexpected results of European matches do result in a stronger market response than expected results, whereas this is not the case in the national competition. Benkraiem, Louhichi and Marques (2009) investigated whether sporting results of European listed football clubs have an impact on stock market (2006-2007). Authors concluded that sport- 
ing results of listed football clubs affect both the abnormal returns and the trading volume around the dates of matches. Also, it was found that the movement and the time when impact occurs depends on the type of result, whether it is defeat, draw or win, and it also depends on match venue, whether it is home or away. Castellani, Pattitoni and Patuelli (2012) analyzed the links between football match results, bets and stock returns of all listed European soccer teams. Sample in this study included all the results and the pre-match betting odds ${ }^{4}$. Results from this study indicate that wins are associated with positive abnormal returns, and ties and losses with negative abnormal returns. Authors also found that role of bets in shaping market reactions to unexpected results are non-significant. Levy (2015) investigated whether sport results for New York City based teams affect daily returns, volatility and trading volume of major stock indexes in the USA (1949-2014). Returns were abnormally high following championships won by New York City professional sports teams, and that returns are abnormally low. Amelie and Darne (2016) focused on 18 countries in the analysis of effects of Fifa World Cup announcements ranging. The sample included both developed and developing countries, with short event windows ranging from -1 to +2 days around the announcement days. Results indicated that losing bidders country stock indices resulted with significant negative CAARs (especially for African countries), whilst winning bidders stock indices did not result with significant results. Torman, Seyhan, Buğan and K1lıç (2016) researched friendship and tournament matches of national football teams of England, Italy, France, Spain and Turkey from year 2002 until 2015. Authors found that football matches of national teams have different impact on stock market and that there is no common effect. Berkowitz and Depken (2018) included financial performance of English football clubs in their analysis. Main result of the research indicates that market asymmetrically responds to winning and losing a match; which is linked to good or bad financial performance of a club.

Other methodology includes regression models of return series and/or GARCH modelling of risk if authors observe effects of match results on risks as well. Here, Berumen and Ceylan (2012) is included, who focused on Chile, UK, Spain and Turkey. Results were interesting in terms that when more successful teams (as in Spain and UK) obtained losing results, investors become more risk averse, but the results were insignificant for winning results. Opposite results were found for less successful teams of Chile and Turkey. Floros (2014) applied the GARCH methodology whilst modelling return and risk series of four football clubs in Europe. The results were somewhat mixed, some return series reacted positively after a win, while some did not react at all. In that way, investors could tailor their strategies more precisely with results from such studies. Turkish national team results were observed in Demirhan (2013). Author found that winning did not affect the stock market index return, whilst losses and draws had negative effects. 
The overall results indicate that in the majority of cases the stock returns react positively to the winning outcomes, and losing causes negative return reactions. Draws have mixed results. It can be said that national sentiment is somewhat affected by the national football team around the matches, in which investors are obviously affected as well, which contributes to the literature on irrational behaviour of investors in certain situations. However, there is some research which did not find any effects. Thus making the research more interesting to see if any effects exist on the Croatian market and how can they be exploited.

\section{Methodology}

Event study methodology is widely known today due to many different applications of stock return (volatility and trading volume, among other) reactions to different economic, political, environmental, social and other events. Thus, this section briefly describes the methodology by following MacKinlay (1997). The actual return of the $i$-th stock on date $t$ is denoted with $r_{i, t}$, return conditioned via some information available on date $t$ is denoted with $E\left(r_{i, t} \mid I\right)$. In order to evaluate if an event had an impact on stock returns, in the first step the abnormal returns $A R_{i, t}$ are calculated as the difference between the actual and conditional return, $A R_{i, t}=r_{i, t}-E\left(r_{i, t} \mid I\right) . I_{t}$ is the information available at date $t$. Conditional return can be calculated as an average return of the pre-event window. However, a more common approach is to estimate a market model in the pre-event window:

$$
E\left(r_{i, t} \mid I\right) \equiv R_{i, t}=\alpha_{i}+\beta_{i} R_{m, t}+\varepsilon_{i, t},
$$

where $R_{m, t}$ denotes the market return at date $t$ and $\varepsilon_{i, t}$ is the error term, $\varepsilon_{i, t} \sim N\left(0, \sigma_{\varepsilon_{i}}^{2}\right)$. If the assumption about the error term does not hold, equation (1) can be estimated with Newey-West (1987) corrections of standard errors or White (1980) heteroskedasticity consistent errors. Additional terms can be added in the equation as well if needed. Usually, the pre-event window length can be set to 250 days (when dealing with daily data). This is why it is opted to include 14 months in the analysis prior to the event window. The second step is to calculate $A R_{i, t}$ in the event window, which is usually a shorter time span, due to short-horizon tests being more powerful (Sheskin 2004). The usual null hypothesis in the test is that the event did not have any effects on stock returns. Several tests are available. Firstly, the average abnormal return can be calculated $A R_{\tau}$ as follows:

$$
\overline{A R}_{\tau}=\frac{1}{N} \sum_{i=1}^{N} A R_{i, \tau},
$$


with variance

$$
\operatorname{var}\left(\overline{A R}_{\tau}\right)=\frac{1}{N^{2}} \sum_{i=1}^{N} \sigma_{\varepsilon_{i}}^{2}
$$

where $N$ denotes number of stocks at date $t$ and $\tau$ denotes a date in the window event. Then, the cumulative abnormal return $\overline{C A R}_{\tau}$ is calculated based upon (2) and its variance as follows:

$$
\overline{C A R}_{\tau}=\sum_{\tau=\tau_{\text {start }}}^{\tau_{\text {end }}} \overline{A R}_{\tau}
$$

and

$$
\operatorname{var}\left(\overline{\mathrm{CAR}}_{\tau}\right)=\frac{1}{N^{2}} \sum_{i=1}^{N} \operatorname{var}\left(\overline{A R}_{\tau}\right)
$$

where $\tau_{\text {start }}$ and $\tau_{\text {end }}$ denote the start and end date of the event window. Finally, the test value is calculated as:

$$
\theta_{1}=\frac{\overline{C A R}_{\tau}}{\sqrt{\operatorname{var}\left(\overline{C A R}_{\tau}\right)}} \sim \mathrm{N}(0,1)
$$

Equivalently, the test statistic can be calculated based upon (4) and (5) as $\theta_{2}=\frac{\overline{A R}_{\tau}}{\sqrt{\operatorname{var}\left(\overline{A R}_{\tau}\right)}} \sim t_{T-d}$ where the degrees of freedom are calculated based upon subtracting the number of independent variables in equation (1) from the time span length of the pre event window. Nonparametric tests include the sign test, in which the null hypothesis is that $p \leq 0.5$, where $p=P\left(C A R_{i} \geq 0\right)$, in which the test statistic is defined as follows:

$$
\theta_{3}=\frac{N^{+}-N}{\sqrt{N}} \sim \mathrm{N}(0,1)^{5},
$$

where $N^{+}$denotes number of positive abnormal returns at date $t$. Wilcoxon signed rank test is another popular nonparametric test in which the test statistic is given by:

$$
\theta_{4}=\sum_{i=1}^{N} R_{i}^{+}
$$

in which $R_{i}^{+}$denotes the positive rank of absolute value of abnormal returns of stock $i$. The test value $\theta_{4}$ for a large $N$ follows a normal distribution with expectation $N(N+1) / 4$ and variance $N(N+1)(2 N+1) / 24$. Corrado (1989) rank test is also a nonparametric test, 
in which abnormal returns are ranked in the estimation and event windows in order to compare the ranks for each stock with the expected average rank. The test statistic follows a unit normal distribution.

The length of the event window is short due to the power of test being greater for shorter windows. Previous literature usually chooses a window length of either several days (5-7) or 21 (-10 up to +10 day). Moreover, the majority of events which are being tested in empirics are assumed to have short term effects. In the case of this research, it chooses the time span as previous related literature -1 up to the +2 day, with the event window being 4 days long. Since there are many matches to be explored in the last couple of years which are included in this study, the time span observed is of course, longer than the pre-event window. But this does not mean that the event window itself is long. It is always equal to 4 days in total. Other details can be found in Serra (2002) on different test and power of tests discussion with respect to sample size can be found in Bartholdy, Olson and Peare (2007).

\section{Empirical results}

\section{Data description}

For the purpose of empirical evaluation of the football results regarding the Croatian national team on the stock returns on Zagreb Stock Exchange, the formal Croatian stock market; daily data on the most liquid stocks has been obtained from ZSE (2018) for the period 2 January 2013 until 1 December 2018. The data includes daily index value of the CROBEX, official stock market index and daily stock prices of the most liquid stocks in year 2017 based upon official statement of ZSE (2017). In that way, the analysis can include more data on the return series in the observed period ${ }^{6}$. In total, data on 60 stocks was collected. Summary statistics is given in table A1 in Appendix. The entire year 2013 and first two months of 2014 were used as the pre-event window in order to estimate the market model given in (1). However, since several matches took place in 2013 as well, the model was augmented as follows:

$$
R_{i, t}=\alpha_{i}+\beta_{i} R_{m, t}+\sum_{i=1}^{I} \gamma_{i} D i_{t}+\beta_{M O N} M^{\prime} N_{t}+\varepsilon_{i},
$$

where $D i_{t}$ is a binary variable equal to unit value the day before a match, the match day and one day after; else it is equal to zero. In that way possible effects of matches which occurred in $2013^{7}$ were excluded. Next, $M O N_{t}$ is a binary variable equal to unit value if the day of the week is Monday in order to capture the weekend effect ${ }^{8}$. In that way, other possible influences on the stock return series which could distort the results are excluded. Environment $R$ package eventstudies (Anand et al. 2017) was used 
for the estimation and calculation part. Equation (9) was estimated by using the least squares method with Newey-West (1987) consistent standard errors for the pre event window. The abnormal returns were then calculated for every event stated in Table 1. Table 1 displays results of football matches of the Croatian national team which were collected from the CFF (Croatian Football Federation) statistics, which is the official page of the Croatian national team. Sample includes 60 matches being played by Croatian national team from year 2014 until 2018. Sample includes friendly, qualification and tournament matches which were played in the given period. In that way, different types of matches were included in the analysis in order to get a more precise picture.

Table 1: Football matches in which Croatian national team participated with dates (event dates) with results.

\begin{tabular}{|c|c|c|c|c|}
\hline Date & Type & Win & Draw & Lose \\
\hline 5 March 2014 & Friendly & & $2-2$ & \\
\hline 31 May 2014 & Friendly & $2-1$ & & \\
\hline 6 June 2014 & Friendly & $1-0$ & & \\
\hline 12 June 2014 & 2014 World cup & & & $3-1$ \\
\hline 18 June 2014 & 2014 World cup & $4-0$ & & \\
\hline 23 June 2014 & 2014 World cup & & & $3-1$ \\
\hline 4 September 2014 & Friendly & $2-0$ & & \\
\hline 9 September 2014 & Euro 2016 qualification & $2-0$ & & \\
\hline 10 October 2014 & Euro 2016 qualification & $1-0$ & & \\
\hline 13 October 2014 & Euro 2016 qualification & $6-0$ & & \\
\hline 12 November 2014 & Friendly & & & $2-1$ \\
\hline 16 November 2014 & Euro 2016 qualification & & $1-1$ & \\
\hline 28 March 2015 & Euro 2016 qualification & $5-1$ & & \\
\hline 7 June 2015 & Friendly & $4-0$ & & \\
\hline 12 June 2015 & Euro 2016 qualification & & $1-1$ & \\
\hline 3 September 2015 & Euro 2016 qualification & & $0-0$ & \\
\hline 6 September 2015 & Euro 2016 qualification & & & $2-0$ \\
\hline 10 October 2015 & Euro 2016 qualification & $3-0$ & & \\
\hline 13 October 2015 & Euro 2016 qualification & $1-0$ & & \\
\hline 17 November 2015 & Friendly & $3-1$ & & \\
\hline 23 March 2016 & Friendly & $2-0$ & & \\
\hline 26 March 2016 & Friendly & & $1-1$ & \\
\hline 27 May 2016 & Friendly & $1-0$ & & \\
\hline 4 June 2016 & Friendly & $10-0$ & & \\
\hline 12 June 2016 & Euro 2016 & $1-0$ & & \\
\hline 17 June 2016 & Euro 2016 & & $2-2$ & \\
\hline 21 June 2016 & Euro 2016 & $2-1$ & & \\
\hline 25 June 2016 & Euro 2016 & & & $1-0$ \\
\hline 5 September 2016 & 2018 World cup qualification & & $1-1$ & \\
\hline 6 October 2016 & 2018 World cup qualification & $6-0$ & & \\
\hline
\end{tabular}


Table 1. Continued

\begin{tabular}{|c|c|c|c|c|}
\hline Date & Type & Win & Draw & Lose \\
\hline 9 October 2016 & 2018 World cup qualification & $1-0$ & & \\
\hline 12 November 2016 & 2018 World cup qualification & $2-0$ & & \\
\hline 15 November 2016 & Friendly & $3-0$ & & \\
\hline 24 March 2017 & 2018 World cup qualification & $1-0$ & & \\
\hline 28 March 2017 & Friendly & & & $3-0$ \\
\hline 27 May 2017 & Friendly & $2-1$ & & \\
\hline 11 June 2017 & 2018 World cup qualification & & & $1-0$ \\
\hline 3 September 2017 & 2018 World cup qualification & $1-0$ & & \\
\hline 5 September 2017 & 2018 World cup qualification & & & $1-0$ \\
\hline 6 October 2017 & 2018 World cup qualification & & $1-1$ & \\
\hline 9 October 2017 & 2018 World cup qualification & $2-0$ & & \\
\hline 9 November 2017 & 2018 World cup qualification & $4-1$ & & \\
\hline 12 November 2017 & 2018 World cup qualification & & $0-0$ & \\
\hline 23 March 2018 & Friendly & & & $2-0$ \\
\hline 27 March 2018 & Friendly & $1-0$ & & \\
\hline 3 June 2018 & Friendly & & & $2-0$ \\
\hline 8 June 2018 & Friendly & $2-1$ & & \\
\hline 16 June 2018 & 2018 World cup & $2-0$ & & \\
\hline 21 June 2018 & 2018 World cup & $3-0$ & & \\
\hline 26 June 2018 & 2018 World cup & $2-1$ & & \\
\hline 1 July 2018 & 2018 World cup & $2-1$ & & \\
\hline 7 July 2018 & 2018 World cup & $3-2$ & & \\
\hline 11 July 2018 & 2018 World cup & $2-1$ & & \\
\hline 15 July 2018 & 2018 World cup & & & $4-2$ \\
\hline 6 September 2018 & Friendly & & $1-1$ & \\
\hline 11 September 2018 & 18/19 Nations League & & & $6-0$ \\
\hline 12 October 2018 & 18/19 Nations League & & $0-0$ & \\
\hline 15 October 2018 & Friendly & $2-1$ & & \\
\hline 15 November 2018 & 18/19 Nations League & $3-2$ & & \\
\hline 18 November 2018 & 18/19 Nations League & & & $2-1$ \\
\hline
\end{tabular}

Source: http://hns-cff.hr/info/statistika/

\section{Event study results}

Firstly, the aggregate results are observed by dividing the games into win, draw and lose as previous literature does. Table 2 shows the cumulative abnormal returns (CAAR) around the date of matches with the test values defined in (6) and (7) with corresponding $p$-values. Day -1 corresponds to the date before the match, day 0 corresponds to date of match, and days 1 and 2 correspond to first and second day after the match took place. In that way, anticipation effects were observed if they were present in the return series (-1 and 0 ) and, as usual, 1-2 days after the match in order 
to observe the short term effects, if they even exist. Previous studies observe only 1 or 2 days after the match, due to explanations that these effects are irrational and short termed. That is why only those two days were observed. Table 2 reveals that neither win, draw nor lose of the Croatian national team has significant impact on the stock price reaction on Zagreb Stock Exchange in the given event window. This is confirmed for both test statistics. It seems that investors in Croatia are not affected by the sporting results of football matches in the observed period. Moreover, the winning matches seem to result with lower CAAR on day +1 compared to the match day (0.0011 compared to previously 0.0012), however it is not significant. Similar conclusions arise for draw and lose matches as well.

Table 2: Event study results of football match results of Croatian national team on stock returns on Zagreb Stock Exchange, summary

\begin{tabular}{|c|c|c|c|c|c|c|c|c|c|}
\hline \multirow{2}{*}{$\boldsymbol{t}$} & \multicolumn{3}{|c|}{ Win } & \multicolumn{3}{c|}{ Draw } & \multicolumn{3}{c|}{ Lose } \\
\cline { 2 - 10 } & $\mathbf{C A A R}_{t}$ & $\boldsymbol{\theta}_{\mathbf{1}}$ & $\boldsymbol{\theta}_{3}$ & $\mathbf{C A A R} \mathbf{R}_{\boldsymbol{t}}$ & $\boldsymbol{\theta}_{1}$ & $\boldsymbol{\theta}_{3}$ & CAAR $_{t}$ & $\boldsymbol{\theta}_{1}$ & $\boldsymbol{\theta}_{3}$ \\
\hline-1 & 0.0014 & $\begin{array}{c}0.043 \\
(0.517)\end{array}$ & $\begin{array}{c}0.023 \\
(0.509)\end{array}$ & -0.0007 & $\begin{array}{c}-0.027 \\
(0.489)\end{array}$ & $\begin{array}{c}0.039 \\
(0.516)\end{array}$ & 0.0009 & $\begin{array}{c}0.043 \\
(0.517)\end{array}$ & $\begin{array}{c}0.035 \\
(0.514)\end{array}$ \\
\hline 0 & 0.0012 & $\begin{array}{c}0.028 \\
(0.511)\end{array}$ & $\begin{array}{c}0.022 \\
(0.509)\end{array}$ & 0.0033 & $\begin{array}{c}0.085 \\
(0.534)\end{array}$ & $\begin{array}{c}0.040 \\
(0.516)\end{array}$ & -0.0013 & $\begin{array}{c}0.034 \\
(0.513)\end{array}$ & $\begin{array}{c}0.033 \\
(0.513)\end{array}$ \\
\hline \multirow{2}{*}{1} & 0.0011 & $\begin{array}{c}0.022 \\
(0.509)\end{array}$ & $\begin{array}{c}0.022 \\
(0.509)\end{array}$ & 0.0026 & $\begin{array}{c}0.048 \\
(0.519)\end{array}$ & $\begin{array}{c}0.047 \\
(0.519)\end{array}$ & -0.0016 & $\begin{array}{c}-0.041 \\
(0.484)\end{array}$ & $\begin{array}{c}0.030 \\
(0.512)\end{array}$ \\
\hline 2 & 0.0023 & $\begin{array}{c}0.039 \\
(0.515)\end{array}$ & $\begin{array}{c}0.022 \\
(0.509)\end{array}$ & 0.0005 & $\begin{array}{c}0.008 \\
(0.503)\end{array}$ & $\begin{array}{c}0.041 \\
(0.516)\end{array}$ & -0.0034 & $\begin{array}{c}-0.066 \\
(0.474)\end{array}$ & $\begin{array}{c}0.030 \\
(0.512)\end{array}$ \\
\hline
\end{tabular}

Note: p-values are given in brackets.

In order to observe if any match had any significant effects on the return series, all of the matches were divided into winning ones, draws and losses and for every date in Table 1 conducted the event study approach. Detailed results are provided in Tables 3, 4 and 5. Again, the conclusions are the same as before. No significant effects (in all tables) were found for any date regardless if the match was winning, draw or losing one. Some CAARs were even lower on day +1 for the winning matches, as some were greater compared to match day for losses. In that way, the results seem to confirm that on the Zagreb Stock Exchange, the investor sentiment is not affected by sporting events in football. However, these results were tested by doing a robustness check in the next section. 
Table 3: Event study results of football match results of Croatian national team on stock returns on Zagreb Stock Exchange, by each event for WIN

\begin{tabular}{|c|c|c|c|c|}
\hline Date & $t$ & $\mathbf{C A A R}_{t}$ & $\boldsymbol{\theta}_{1}$ & $\boldsymbol{\theta}_{3}$ \\
\hline \multirow{4}{*}{31 May 2014} & -1 & -0.0017 & $-0.062(0.475)$ & $0.104(0.541)$ \\
\hline & 0 & -0.0089 & $-0.159(0.437)$ & $0.094(0.537)$ \\
\hline & 1 & -0.0025 & $-0.039(0.485)$ & $0.125(0.550)$ \\
\hline & 2 & 0.0216 & $0.253(0.600)$ & $0.156(0.562)$ \\
\hline \multirow{5}{*}{6 June 2014} & -1 & 0.0149 & $0.423(0.664)$ & $0.185(0.574)$ \\
\hline & 0 & 0.0206 & $0.362(0.641)$ & $0.171(0.568)$ \\
\hline & 1 & 0.0191 & $0.248(0.598)$ & $0.128(0.551)$ \\
\hline & 2 & 0.0199 & $0.246(0.597)$ & $0.171(0.568)$ \\
\hline & -1 & 0.0041 & $0.173(0.568)$ & $0.125(0.550)$ \\
\hline \multirow[t]{3}{*}{18 June 2014} & 0 & -0.0019 & $-0.071(0.472)$ & $0.104(0.541)$ \\
\hline & 1 & -0.0028 & $-0.064(0.475)$ & $0.104(0.541)$ \\
\hline & 2 & 0.0006 & $0.013(0.505)$ & $0.125(0.550)$ \\
\hline \multirow{4}{*}{4 September 2014} & -1 & 0.0002 & $0.009(0.504)$ & $0.078(0.531)$ \\
\hline & 0 & -0.0028 & $-0.107(0.458)$ & $0.034(0.568)$ \\
\hline & 1 & 0.0028 & $0.065(0.526)$ & $0.067(0.527)$ \\
\hline & 2 & 0.0055 & $0.102(0.540)$ & $0.078(0.531)$ \\
\hline \multirow{4}{*}{9 September 2014} & -1 & 0.0056 & $0.256(0.601)$ & $0.109(0.543)$ \\
\hline & 0 & 0.0077 & $0.224(0.589)$ & $0.090(0.536)$ \\
\hline & 1 & 0.0140 & $0.295(0.616)$ & $0.109(0.543)$ \\
\hline & 2 & 0.0172 & $0.379(0.648)$ & $0.115(0.546)$ \\
\hline \multirow{4}{*}{10 October 2014} & -1 & -0.0106 & $-0.507(0.306)$ & $0.045(0.518)$ \\
\hline & 0 & -0.0282 & $-0.830(0.203)$ & $0.036(0.514)$ \\
\hline & 1 & -0.0501 & $-1.035(0.150)$ & $0.027(0.511)$ \\
\hline & 2 & -0.0508 & $-1.124(0.131)$ & $0.009(0.504)$ \\
\hline \multirow{4}{*}{13 October 2014} & -1 & -0.0177 & $-0.669(0.252)$ & $0.045(0.518)$ \\
\hline & 0 & -0.0396 & $-1.003(0.158)$ & $0.036(0.514)$ \\
\hline & 1 & -0.0402 & $-1.098(0.136)$ & 0.009 (0.504) \\
\hline & 2 & -0.0600 & $-1.316(0.094)$ & $0.009(0.504)$ \\
\hline \multirow{4}{*}{28 March 2015} & -1 & -0.0029 & $-0.161(0.436)$ & $0.103(0.541)$ \\
\hline & 0 & -0.0004 & $-0.019(0.493)$ & $0.069(0.527)$ \\
\hline & 1 & 0.0038 & $0.088(0.535)$ & $0.086(0.534)$ \\
\hline & 2 & 0.0163 & $0.251(0.599)$ & $0.138(0.555)$ \\
\hline \multirow{4}{*}{7 June 2015} & -1 & -0.0091 & $-0.476(0.317)$ & $0.086(0.534)$ \\
\hline & 0 & -0.0031 & $-0.108(0.457)$ & $0.120(0.548)$ \\
\hline & 1 & -0.0149 & $-0.465(0.321)$ & $0.138(0.555)$ \\
\hline & 2 & -0.0365 & $-0.458(0.324)$ & $0.086(0.534)$ \\
\hline \multirow{4}{*}{10 October 2015} & -1 & 0.0059 & $0.112(0.545)$ & $0.063(0.525)$ \\
\hline & 0 & -0.0021 & $-0.072(0.471)$ & $0.094(0.537)$ \\
\hline & 1 & -0.0024 & $-0.076(0.470)$ & $0.094(0.537)$ \\
\hline & 2 & 0.0025 & $0.069(0.527)$ & $0.094(0.537)$ \\
\hline \multirow{4}{*}{13 October 2015} & -1 & -0.0011 & $-0.052(0.479)$ & $0.141(0.556)$ \\
\hline & 0 & 0.0045 & $0.251(0.599)$ & $0.125(0.550)$ \\
\hline & 1 & -0.0152 & $-0.331(0.370)$ & $0.109(0.544)$ \\
\hline & 2 & -0.0134 & $-0.304(0.381)$ & $0.094(0.537)$ \\
\hline
\end{tabular}




\section{Table 3. Continued}

\begin{tabular}{|c|c|c|c|c|}
\hline Date & $t$ & $\mathbf{C A A R}_{t}$ & $\boldsymbol{\theta}_{1}$ & $\boldsymbol{\theta}_{3}$ \\
\hline \multirow{4}{*}{17 November 2015} & -1 & $\begin{array}{l}-0.0090 \\
\end{array}$ & $-0.531(0.298)$ & $0.107(0.542)$ \\
\hline & 0 & -0.0040 & $-0.160(0.436)$ & $0.085(0.534)$ \\
\hline & 1 & -0.0006 & $-0.021(0.492)$ & $0.128(0.551)$ \\
\hline & 2 & 0.0043 & $0.133(0.553)$ & $0.128(0.551)$ \\
\hline \multirow{4}{*}{23 March 2016} & -1 & 0.0069 & $0.598(0.725)$ & $0.210(0.583)$ \\
\hline & 0 & 0.0123 & $0.614(0.730)$ & $0.229(0.591)$ \\
\hline & 1 & 0.0131 & $0.670(0.748)$ & $0.191(0.576)$ \\
\hline & 2 & 0.0145 & $0.521(0.699)$ & $0.210(0.583)$ \\
\hline \multirow{4}{*}{27 May 2016} & -1 & 0.0023 & $0.124(0.549)$ & $0.153(0.561)$ \\
\hline & 0 & 0.0028 & $0.133(0.553)$ & $0.134(0.553)$ \\
\hline & 1 & 0.0030 & $0.147(0.559)$ & $0.095(0.538)$ \\
\hline & 2 & 0.0071 & $0.277(0.609)$ & $0.134(0.553)$ \\
\hline \multirow{4}{*}{4 June 2016} & -1 & -0.0003 & $-0.009(0.497)$ & $0.177(0.570)$ \\
\hline & 0 & -0.0263 & $-0.434(0.332)$ & $0.133(0.553)$ \\
\hline & 1 & -0.0365 & $-0.707(0.240)$ & $0.088(0.535)$ \\
\hline & 2 & -0.0455 & $-0.811(0.209)$ & $0.044(0.518)$ \\
\hline \multirow{4}{*}{12 June 2016} & -1 & 0.0087 & $0.904(0.817)$ & 0.270 (0.606) \\
\hline & 0 & 0.0109 & $0.547(0.708)$ & $0.216(0.585)$ \\
\hline & 1 & 0.0061 & $0.538(0.705)$ & $0.270(0.606)$ \\
\hline & 2 & 0.0075 & $0.852(0.803)$ & $0.270(0.606)$ \\
\hline \multirow{4}{*}{21 June 2016} & -1 & -0.0057 & $-0.207(0.418)$ & $0.158(0.563)$ \\
\hline & 0 & 0.0080 & $0.468(0.680)$ & $0.221(0.588)$ \\
\hline & 1 & 0.0015 & $0.109(0.544)$ & $0.158(0.563)$ \\
\hline & 2 & -0.0165 & $-0.991(0.161)$ & $0.032(0.513)$ \\
\hline \multirow{4}{*}{6 October 2016} & -1 & 0.0039 & $0.203(0.581)$ & $0.207(0.582)$ \\
\hline & 0 & 0.0098 & $0.375(0.646)$ & $0.138(0.555)$ \\
\hline & 1 & 0.0174 & $0.556(0.711)$ & $0.155(0.562)$ \\
\hline & 2 & 0.0300 & $0.582(0.720)$ & $0.189(0.575)$ \\
\hline \multirow{4}{*}{9 October 2016} & -1 & 0.0077 & $0.403(0.657)$ & $0.141(0.556)$ \\
\hline & 0 & 0.0179 & $0.521(0.699)$ & $0.141(0.556)$ \\
\hline & 1 & 0.0309 & $0.534(0.703)$ & $0.156(0.562)$ \\
\hline & 2 & 0.0293 & $0.443(0.671)$ & $0.125(0.550)$ \\
\hline \multirow{4}{*}{12 November 2016} & -1 & 0.0264 & $0.846(0.801)$ & $0.241(0.595)$ \\
\hline & 0 & 0.0382 & $1.001(0.842)$ & $0.241(0.595)$ \\
\hline & 1 & 0.0539 & $0.915(0.820)$ & $0.241(0.595)$ \\
\hline & 2 & 0.0597 & $0.671(0.749)$ & $0.224(0.589)$ \\
\hline \multirow{4}{*}{15 November 2016} & -1 & 0.0147 & $0.441(0.670)$ & $0.143(0.557)$ \\
\hline & 0 & 0.0188 & $0.278(0.609)$ & $0.114(0.545)$ \\
\hline & 1 & 0.0175 & $0.269(0.606)$ & $0.128(0.551)$ \\
\hline & 2 & 0.0177 & $0.289(0.614)$ & $0.128(0.551)$ \\
\hline \multirow{4}{*}{24 March 2017} & -1 & -0.0299 & $-1.146(0.126)$ & $0.011(0.504)$ \\
\hline & 0 & -0.0369 & $-1.221(0.111)$ & $0.011(0.504)$ \\
\hline & 1 & -0.0090 & $-0.407(0.342)$ & $0.078(0.531)$ \\
\hline & 2 & -0.0018 & $-0.052(0.479)$ & $0.078(0.531)$ \\
\hline \multirow{4}{*}{27 May 2017} & -1 & 0.0039 & $0.191(0.576)$ & $0.082(0.533)$ \\
\hline & 0 & 0.0078 & $0.484(0.686)$ & $0.219(0.587)$ \\
\hline & 1 & 0.0045 & $0.156(0.562)$ & $0.192(0.576)$ \\
\hline & 2 & 0.0083 & $0.288(0.613)$ & $0.164(0.565)$ \\
\hline
\end{tabular}


Table 3. Continued

\begin{tabular}{|c|c|c|c|c|}
\hline Date & $t$ & CAAR $_{t}$ & $\theta_{1}$ & $\boldsymbol{\theta}_{3}$ \\
\hline \multirow{4}{*}{3 September 2017} & -1 & 0.0035 & $0.241(0.595)$ & $0.126(0.550)$ \\
\hline & 0 & -0.0025 & $-0.169(0.433)$ & $0.095(0.538)$ \\
\hline & 1 & -0.0049 & $-0.244(0.404)$ & $0.063(0.525)$ \\
\hline & 2 & -0.0070 & $-0.321(0.374)$ & $0.095(0.538)$ \\
\hline \multirow{4}{*}{9 October 2017} & -1 & 0.0083 & $0.864(0.806)$ & $0.309(0.621)$ \\
\hline & 0 & 0.0067 & $0.657(0.744)$ & $0.265(0.605)$ \\
\hline & 1 & 0.0095 & $0.908(0.818)$ & $0.309(0.621)$ \\
\hline & 2 & 0.0238 & $1.201(0.885)$ & $0.309(0.621)$ \\
\hline \multirow{4}{*}{9 November 2017} & -1 & 0.0127 & $0.579(0.719)$ & $0.229(0.591)$ \\
\hline & 0 & 0.0209 & $0.524(0.700)$ & $0.210(0.583)$ \\
\hline & 1 & 0.0296 & $0.695(0.757)$ & $0.248(0.598)$ \\
\hline & 2 & 0.0234 & $0.455(0.675)$ & $0.229(0.591)$ \\
\hline \multirow{4}{*}{27 March 2018} & -1 & 0.0066 & $0.418(0.662)$ & $0.168(0.567)$ \\
\hline & 0 & 0.0027 & $0.166(0.566)$ & $0.192(0.576)$ \\
\hline & 1 & -0.0089 & $-0.237(0.406)$ & $0.096(0.538)$ \\
\hline & 2 & -0.0105 & $-0.311(0.378)$ & $0.072(0.529)$ \\
\hline \multirow{4}{*}{8 June 2018} & -1 & -0.0185 & $-0.461(0.322)$ & $0.133(0553)$ \\
\hline & 0 & -0.0137 & $-0.405(0.343)$ & $0.265(0.605)$ \\
\hline & 1 & -0.0202 & $-0.551(0.291)$ & $0.133(0.553)$ \\
\hline & 2 & -0.0228 & $-0.546(0.293)$ & $0.088(0.535)$ \\
\hline \multirow{4}{*}{16 June 2018} & -1 & 0.0005 & $0.031(0.512)$ & $0.133(0.553)$ \\
\hline & 0 & -0.0028 & $-0.216(0.415)$ & $0.088(0.535)$ \\
\hline & 1 & -0.0145 & $-0.879(0.190)$ & $0.044(0.518)$ \\
\hline & 2 & -0.0091 & $-0.493(0.311)$ & $0.088(0.535)$ \\
\hline \multirow{4}{*}{21 June 2018} & -1 & 0.0019 & $0.251(0.599)$ & $0.137(0.555)$ \\
\hline & 0 & 0.0136 & $1.062(0.856)$ & $0.247(0.597)$ \\
\hline & 1 & -0.0005 & $-0.051(0.480)$ & $0.137(0.555)$ \\
\hline & 2 & 0.0017 & $0.065(0.526)$ & $0.110(0.544)$ \\
\hline \multirow{4}{*}{26 June 2018} & -1 & 0.0102 & $0.784(0.784)$ & $0.221(0.588)$ \\
\hline & 0 & -0.0028 & $-0.345(0.365)$ & $0.095(0.538)$ \\
\hline & 1 & -0.0059 & $-0.444(0.328)$ & $0.095(0.538)$ \\
\hline & 2 & -0.0128 & $-0.535(0.296)$ & $0.063(0.525)$ \\
\hline \multirow{4}{*}{1 July 2018} & -1 & -0.0059 & $-0.381(0.352)$ & $0.185(0.573)$ \\
\hline & 0 & -0.0016 & $-0.126(0.450)$ & $0.185(0.573)$ \\
\hline & 1 & -0.0045 & $-0.316(0.376)$ & $0.185(0.573)$ \\
\hline & 2 & -0.0065 & $-0.401(0.344)$ & $0.111(0.544)$ \\
\hline \multirow{4}{*}{7 July 2018} & -1 & 0.0095 & $0.501(0.692)$ & $0.270(0.606)$ \\
\hline & 0 & 0.0049 & $0.519(0.698)$ & $0.270(0.606)$ \\
\hline & 1 & 0.0087 & $0.841(0.800)$ & $0.324(0.627)$ \\
\hline & 2 & 0.0137 & $0.833(0.798)$ & $0.378(0.647)$ \\
\hline \multirow{4}{*}{11 July 2018} & -1 & 0.0037 & $0.885(0.812)$ & $0.358(0.640)$ \\
\hline & 0 & 0.0024 & $0.364(0.642)$ & $0.358(0.640)$ \\
\hline & 1 & 0.0003 & $0.047(0.519)$ & $0.268(0.606)$ \\
\hline & 2 & 0.0006 & $0.111(0.544)$ & $0.179(0.571)$ \\
\hline
\end{tabular}


Table 3. Continued

\begin{tabular}{|c|c|c|c|c|}
\hline Date & $\boldsymbol{t}$ & $\mathbf{C A A R}_{t}$ & $\boldsymbol{\theta}_{1}$ & $\boldsymbol{\theta}_{3}$ \\
\hline \multirow{3}{*}{15 October 2018 } & -1 & 0.0128 & $0.258(0.602)$ & $0.219(0.587)$ \\
\cline { 2 - 5 } & 0 & 0.0325 & $0.296(0.616)$ & $0.192(0.576)$ \\
\cline { 2 - 5 } & 1 & 0.0411 & $0.391(0.652)$ & $0.192(0.576)$ \\
\cline { 2 - 5 } & 2 & 0.0491 & $0.458(0.677)$ & $0.192(0.576)$ \\
\cline { 2 - 5 } 15 November 2018 & -1 & 0.0169 & $0.144(0.557)$ & $0.048(0.519)$ \\
\cline { 2 - 5 } & 0 & 0.0291 & $0.244(0.596)$ & $0.096(0.538)$ \\
\cline { 2 - 5 } & 1 & 0.0121 & $0.208(0.582)$ & $0.144(0.557)$ \\
\hline
\end{tabular}

Note: p-values are given in brackets.

Table 4: Event study results of football match results of Croatian national team on stock returns on Zagreb Stock Exchange, by each event for DRAW

\begin{tabular}{|c|c|c|c|c|}
\hline Date & $t$ & CAAR $_{t}$ & $\theta_{1}$ & $\theta_{3}$ \\
\hline \multirow{4}{*}{5 March 2014} & -1 & -0.0161 & $-0.659(0.255)$ & $0.058(0.523)$ \\
\hline & 0 & -0.0028 & $-0.063(0.475)$ & $0.097(0.539)$ \\
\hline & 1 & 0.0079 & $0.141(0.556)$ & $0.126(0.550)$ \\
\hline & 2 & 0.0087 & $0.145(0.558)$ & $0.126(0.550)$ \\
\hline \multirow{4}{*}{16 November 2014} & -1 & -0.0003 & $-0.025(0.490)$ & $0.118(0.547)$ \\
\hline & 0 & -0.0039 & $-0.222(0.412)$ & $0.105(0.542)$ \\
\hline & 1 & -0.0082 & $-0.430(0.333)$ & $0.092(0.537)$ \\
\hline & 2 & -0.0119 & $-0.408(0.342)$ & $0.026(0.510)$ \\
\hline \multirow{4}{*}{12 June 2015} & -1 & 0.0121 & $0.440(0.670)$ & $0.156(0.562)$ \\
\hline & 0 & 0.0157 & $0.403(0.656)$ & $0.125(0.550)$ \\
\hline & 1 & 0.0165 & $0.459(0.677)$ & $0.172(0.568)$ \\
\hline & 2 & 0.0057 & $0.143(0.557)$ & $0.109(0.544)$ \\
\hline \multirow{4}{*}{3 September 2015} & -1 & 0.0051 & $0.211(0.584)$ & $0.128(0.551)$ \\
\hline & 0 & 0.0035 & $0.206(0.582)$ & $0.171(0.568)$ \\
\hline & 1 & 0.0047 & $0.266(0.605)$ & $0.192(0.576)$ \\
\hline & 2 & -0.0034 & $-0.151(0.440)$ & $0.149(0.559)$ \\
\hline \multirow{4}{*}{26 March 2016} & -1 & 0.0059 & $0.346(0.635)$ & $0.153(0.561)$ \\
\hline & 0 & 0.0085 & $0.441(0.670)$ & $0.191(0.576)$ \\
\hline & 1 & 0.0037 & $0.146(0.558)$ & $0.172(0.568)$ \\
\hline & 2 & 0.0034 & $0.101(0.540)$ & $0.191(0.576)$ \\
\hline \multirow{4}{*}{17 June 2016} & -1 & -0.0048 & $-0.785(0.216)$ & $0.108(0.543)$ \\
\hline & 0 & -0.0103 & $-0.510(0.305)$ & $0.108(0.543)$ \\
\hline & 1 & -0.0201 & $-0.386(0.350)$ & $0.270(0.606)$ \\
\hline & 2 & 0.0029 & $0.307(0.621)$ & $0.216(0.585)$ \\
\hline \multirow{4}{*}{5 September 2016} & -1 & -0.0067 & $-0.304(0.380)$ & $0.115(0.546)$ \\
\hline & 0 & -0.0085 & $-0.287(0.387)$ & $0.115(0.546)$ \\
\hline & 1 & -0.0129 & $-0.292(0.385)$ & $0.134(0.553)$ \\
\hline & 2 & -0.0058 & $-0.113(0.455)$ & $0.153(0.561)$ \\
\hline
\end{tabular}


Table 4. Continued

\begin{tabular}{|c|c|c|c|c|}
\hline Date & $\boldsymbol{t}$ & $\mathbf{C A A R}_{\boldsymbol{t}}$ & $\boldsymbol{\theta}_{\mathbf{1}}$ & $\boldsymbol{\theta}_{\mathbf{3}}$ \\
\hline \multirow{4}{*}{6 October 2017 } & -1 & 0.0028 & $0.239(0.595)$ & $0.265(0.605)$ \\
\cline { 2 - 5 } & 0 & 0.0111 & $0.566(0.714)$ & $0.309(0.621)$ \\
\cline { 2 - 5 } & 1 & 0.0095 & $0.642(0.740)$ & $0.309(0.621)$ \\
\hline \multirow{4}{*}{ 12 November 2017 } & 2 & 0.0123 & $1.055(0.557)$ & $0.309(0.621)$ \\
\cline { 2 - 5 } & -1 & 0.0018 & $0.059(0.524)$ & $0.144(0.557)$ \\
\cline { 2 - 5 } & 0 & 0.0026 & $0.058(0.523)$ & $0.131(0.522)$ \\
\cline { 2 - 5 } & 1 & -0.0132 & $-0.163(0.435)$ & $0.118(0.547)$ \\
\cline { 2 - 5 } & 2 & -0.0304 & $-0.274(0.392)$ & $0.105(0.542)$ \\
\cline { 2 - 5 } & -1 & 0.0209 & $0.373(0.645)$ & $0.340(0.633)$ \\
\cline { 2 - 5 } & 0 & 0.0374 & $0.332(0.630)$ & $0.136(0.554)$ \\
\cline { 2 - 5 } & 1 & 0.0279 & $0.271(0.607)$ & $0.204(0.581)$ \\
\hline \multirow{3}{*}{ 12 October 2018 } & 2 & 0.0326 & $0.271(0.607)$ & $0.204(0.581)$ \\
\cline { 2 - 5 } & -1 & -0.0113 & $-0.992(0.161)$ & $0.024(0.510)$ \\
\cline { 2 - 5 } & 0 & 0.0030 & $0.079(0.532)$ & $0.024(0.510)$ \\
\cline { 2 - 5 } & 1 & 0.0249 & $0.256(0.601)$ & $0.096(0.538)$ \\
\hline
\end{tabular}

Note: p-values are given in brackets.

Table 5: Event study results of football match results of Croatian national team on stock returns on Zagreb Stock Exchange, by each event for LOSE

\begin{tabular}{|c|c|c|c|c|}
\hline Date & $t$ & CAAR $_{t}$ & $\theta_{1}$ & $\theta_{3}$ \\
\hline \multirow{4}{*}{12 June 2014} & -1 & 0.0009 & $0.028(0.511)$ & $0.131(0.552)$ \\
\hline & 0 & 0.0082 & $0.177(0.570)$ & $0.144(0.557)$ \\
\hline & 1 & 0.0119 & $0.220(0.587)$ & $0.118(0.547)$ \\
\hline & 2 & 0.0234 & $0.328(0.628)$ & $0.131(0.552)$ \\
\hline \multirow{4}{*}{23 June 2014} & -1 & 0.0000 & $-0.001(0.499)$ & $0.131(0.552)$ \\
\hline & 0 & 0.0025 & $0.093(0.499)$ & $0.118(0.547)$ \\
\hline & 1 & -0.0010 & $-0.031(0.537)$ & $0.131(0.552)$ \\
\hline & 2 & -0.0012 & $-0.031(0.488)$ & $0.105(0.542)$ \\
\hline \multirow{4}{*}{12 November 2014} & -1 & -0.0059 & $-0.265(0.488)$ & $0.078(0.531)$ \\
\hline & 0 & -0.0020 & $-0.096(0.462)$ & $0.097(0.539)$ \\
\hline & 1 & -0.0001 & $-0.002(0.499)$ & $0.097(0.539)$ \\
\hline & 2 & -0.0026 & $-0.070(0.472)$ & $0.097(0.539)$ \\
\hline \multirow{4}{*}{6 September 2015} & -1 & -0.0029 & $-0.210(0.417)$ & $0.120(0.548)$ \\
\hline & 0 & -0.0016 & $-0.110(0.456)$ & $0.144(0.557)$ \\
\hline & 1 & -0.0098 & $-0.459(0.323)$ & $0.096(0.538)$ \\
\hline & 2 & -0.0066 & $-0.237(0.406)$ & $0.144(0.557)$ \\
\hline \multirow{4}{*}{25 June 2016} & -1 & -0.0099 & $-0.378(0.353)$ & $0.111(0.544)$ \\
\hline & 0 & -0.0309 & $-0.973(0.165)$ & $0.000(0.500)$ \\
\hline & 1 & -0.0313 & $-0.821(0.206)$ & $0.000(0.500)$ \\
\hline & 2 & -0.0158 & $-1.049(0.147)$ & $0.037(0.515)$ \\
\hline
\end{tabular}


Table 5. Continued

\begin{tabular}{|c|c|c|c|c|}
\hline Date & $t$ & $\mathbf{C A A R}_{t}$ & $\theta_{1}$ & $\theta_{3}$ \\
\hline \multirow{4}{*}{28 March 2017} & -1 & 0.0085 & $0.439(0.670)$ & $0.126(0.550)$ \\
\hline & 0 & 0.0021 & $0.064(0.525)$ & $0.087(0.535)$ \\
\hline & 1 & -0.0214 & $-0.432(0.333)$ & $0.029(0.512)$ \\
\hline & 2 & -0.0532 & $-1.097(0.136)$ & $0.019(0.508)$ \\
\hline \multirow{4}{*}{11 June 2017} & -1 & 0.0072 & $0.301(0.618)$ & $0.155(0.562)$ \\
\hline & 0 & 0.0267 & $0.400(0.655)$ & $0.189(0.575)$ \\
\hline & 1 & 0.0382 & $0.558(0.712)$ & $0.207(0.582)$ \\
\hline & 2 & 0.0417 & $0.496(0.690)$ & $0.207(0.582)$ \\
\hline \multirow{4}{*}{5 September 2017} & -1 & 0.0009 & $0.048(0.519)$ & $0.137(0.555)$ \\
\hline & 0 & -0.0016 & $-0.070(0.472)$ & $0.110(0.544)$ \\
\hline & 1 & -0.0042 & $-0.151(0.440)$ & $0.137(0.555)$ \\
\hline & 2 & -0.0046 & $-0.109(0.456)$ & $0.110(0.544)$ \\
\hline \multirow{4}{*}{23 March 2018} & -1 & -0.0028 & $-0.233(0.408)$ & $0.076(0.530)$ \\
\hline & 0 & -0.0100 & $-0.538(0.295)$ & $0.057(0.523)$ \\
\hline & 1 & -0.0059 & $-0.329(0.371)$ & $0.076(0.530)$ \\
\hline & 2 & -0.0107 & $-0.605(0.273)$ & $0.076(0.530)$ \\
\hline \multirow{4}{*}{3 June 2018} & -1 & -0.0021 & $-0.238(0.406)$ & $0.110(0.544)$ \\
\hline & 0 & 0.0007 & $0.021(0.508)$ & $0.137(0.555)$ \\
\hline & 1 & -0.0002 & $-0.004(0.499)$ & $0.110(0.544)$ \\
\hline & 2 & 0.0029 & $0.077(0.531)$ & $0.110(0.544)$ \\
\hline \multirow{4}{*}{15 July 2018} & -1 & -0.0021 & $-0.231(0.409)$ & $0.268(0.606)$ \\
\hline & 0 & -0.0018 & $-0.274(0.392)$ & $0.268(0.606)$ \\
\hline & 1 & -0.0034 & $-0.438(0.331)$ & $0.179(0.571)$ \\
\hline & 2 & -0.0049 & $-0.414(0.339)$ & $0.179(0.571)$ \\
\hline \multirow{4}{*}{11 September 2018} & -1 & -0.0004 & $-0.020(0.492)$ & $0.177(0.570)$ \\
\hline & 0 & 0.0217 & $0.211(0.584)$ & $0.088(0.535)$ \\
\hline & 1 & 0.0157 & $0.263(0.604)$ & $0.221(0.587)$ \\
\hline & 2 & 0.0355 & $0.364(0.642)$ & $0.221(0.587)$ \\
\hline \multirow{4}{*}{18 November 2018} & -1 & 0.0135 & $0.377(0.647)$ & $0.164(0.565)$ \\
\hline & 0 & -0.0042 & $-0.062(0.475)$ & $0.219(0.587)$ \\
\hline & 1 & -0.0113 & $-0.205(0.419)$ & $0.164(0.565)$ \\
\hline & 2 & -0.0219 & $-0.256(0.399)$ & $0.164(0.565)$ \\
\hline
\end{tabular}

Note: p-values are given in brackets.

\section{Robustness checking}

Following Edmans et al. (2007), CAARs for ever match on day +1 were obtained, i.e. after the match day and by using panel regression estimated the equation in which CAARs depend upon the binary variables of winning, losing or having a draw match:

$$
C A A R_{i, t}=w_{i} W_{i, t}+d_{i} D R A W_{i, t}+l_{i} \operatorname{LOSE}_{i, t}+\varepsilon_{i, t},
$$


where $W I N_{i, t}$ is equal to 1 for winning, else $0 ; D R A W_{i, t}$ equal to unit value for a draw match, else 0 and $L O S E_{i, t}$ equal to 1 for losing on match day. The results are shown in Table 6. It can be seen that no significant results were found in all of the outcomes. This is not consider being economically significant due to detailed results in previous section, as well as the small coefficient of determination confirms that match results do not explain return movements on ZSE on day after the match. The coefficient besides LOSE variable is of greater magnitude and negative as well; as in Edmans et al. (2007).

Table 6: Panel regression results for win, draw and lose

\begin{tabular}{|c|c|c|c|c|}
\hline WIN & DRAW & LOSE & $\mathbf{R}^{2}$ & $\mathbf{N}$ \\
\hline$-0.003(0.2534)$ & $0.0053(0.1119)$ & $\begin{array}{c}-0.0045 \\
(0.1311)\end{array}$ & 0.0067 & 825 \\
\hline
\end{tabular}

Note: p-values are given in brackets and are calculated based upon panel-corrected standard errors.

Finally, in order to see if any effects could be found by dividing the matches into qualification ones, friendly and competitive tournaments, the sample is divided into those three categories. Thus, equation (10) was re-estimated for the mentioned categories. Results of the panel regression estimation are shown in Table 7. As previously, no significant effects were found for any of the match outcomes.

Table 7: Panel regression results for qualification, friendly games and tournaments

\begin{tabular}{|c|c|c|c|}
\hline Type of game: & Qualification & Friendly & Competitive tournament \\
\hline \multirow{2}{*}{ WIN } & -0.014 & -0.007 & -0.003 \\
& $(0.1113)$ & $(0.4259)$ & $(0.5496)$ \\
\hline \multirow{2}{*}{ DRAW } & -0.002 & 0.009 & 0.008 \\
& $(0.7934)$ & $(0.5888)$ & $(0.4803)$ \\
\hline \multirow{2}{*}{ LOSE } & -0.008 & -0.008 & -0.001 \\
& $(0.4129)$ & $(0.5786)$ & $(0.8110)$ \\
\hline $\mathrm{R}^{2}$ & 0.005 & 0.002 & 0.004 \\
\hline $\mathrm{N}$ & 344 & 292 & 189 \\
\hline
\end{tabular}

Note: p-values are given in brackets and are calculated based upon panel-corrected standard errors.

\section{Expectations augmented model}

As previous literature suggests (see Edmans et al. 2007, Palomino et al. 2005), the results should be adjusted for the pre-match expectations of a given result. That is why data on betting odds for every match observed in this study was collected from oddsportal (2019) for winning, draw and losing and calculated the probability $p_{i, t}$ of the national team to win as suggested in Bernile and Lyandres (2011): 


$$
p_{i, t}=\frac{1 / O_{\text {win }, i, t}}{1 / O_{\text {win }, i, t}+1 / O_{\text {draw }, i, t}+1 / O_{\text {lose }, i, t}},
$$

where $O_{\text {win }, i, t}, O_{\text {draw }, i, t}$ and $O_{\text {lose }, i, t}$ denote bookmaker odds of winning, draw game or losing (for national team $i$ and date $t$ ). In the second step, event study estimations as in Tables 2-5 were re-done with inclusion of the probability $p_{i, t}$ in the market model for the day after the match. The new results are shown in Tables A2-A5 in the Appendix. The results remained the same. Finally, as in Edmans et al. (2007), the model in which the CAARs on day +1 are dependent upon the binary variable of winning (previously denoted WIN) and the probability defined in (10) were estimated as well:

$$
C A A R_{i, t}=\alpha_{0}+\alpha_{1} W I N_{i, t}+\alpha_{2} p_{i, t}+\varepsilon_{i, t},
$$

where it should hold that $\alpha_{0}$ is not significant, $\alpha_{1}>0$ and $\alpha_{2}=-\alpha_{1}<0$. This is due to the explanation that rational investor should price the loss effect stronger for losses which were unexpected. This is easily seen if the probability of winning $p_{i, t}$ is high but the team loses (i.e. the WIN binary is equal to 0 ), this should be negatively reflected in CAARs. The results of estimating (12) on the full sample and subsamples are shown in Table 8. Again, the results are not significant for both variables in the model, WIN and probability; meaning that investors on the ZSE do not take into account football events and results when pricing the stocks. Finally, model (12) was re-estimated with the restriction that $\alpha_{2}=-\alpha_{1}$. Results given in Table 9, again, show the same result as previously mentioned. Thus, no effects on stock returns could be found for the examined matches in the observed period, even when controlling for expectations.

Table 8: Panel regression total results; results for qualification, friendly games and tournaments, expectations included

\begin{tabular}{|c|c|c|c|c|}
\hline Type of game: & Total & Qualification & Friendly & Competitive tournament \\
\hline$\hat{\alpha}_{0}$ & -0.001 & -0.003 & 0.002 & -0.003 \\
& $(0.695)$ & $(0.520)$ & $(0.503)$ & $(0.679)$ \\
\hline$\hat{\alpha}_{1}$ & -0.002 & -0.0002 & -0.001 & -0.008 \\
& $(0.299)$ & $(0.953)$ & $(0.818)$ & $(0.120)$ \\
\hline$\hat{\alpha}_{2}$ & 0.002 & 0.002 & -0.003 & 0.011 \\
$\mathrm{R}^{2}$ & $(0.654)$ & $(0.762)$ & $(0.716)$ & $(0.483)$ \\
\hline
\end{tabular}

Note: p-values are given in brackets and are calculated based upon panel-corrected standard errors. 
Table 9: Panel regression total results; results for qualification, friendly games and tournaments, expectations included, restrictions included

\begin{tabular}{|c|c|c|c|c|}
\hline Type of game: & Total & Qualification & Friendly & Competitive tournament \\
\hline \multirow{2}{*}{$\hat{\alpha}_{0}$} & -0.001 & -0.002 & 0.0005 & -0.002 \\
& $(0.287)$ & $(0.253)$ & $(0.799)$ & $(0.557)$ \\
\hline$\hat{\alpha}_{1}$ & -0.002 & $-7 \cdot 10^{-5}$ & -0.001 & -0.008 \\
& $(0.298)$ & $(0.980)$ & $(0.839)$ & $(0.127)$ \\
\hline$\hat{\alpha}_{2}$ & 0.002 & $7 \cdot 10^{-5}$ & 0.001 & 0.008 \\
\hline $\mathrm{R}^{2}$ & $(0.298)$ & $(0.980)$ & $(0.839)$ & $(0.127)$ \\
\hline
\end{tabular}

Note: p-values are given in brackets and are calculated based upon panel-corrected standard errors.

\section{Discussion}

The results in the empirical analysis indicate that sporting outcomes regarding football matches did not affect the investor sentiment on the Croatian stock market. These results are in line with some of the previous literature in which authors did not find any effects as well (see literature review section). The conclusion stays the same even when controlling for expectations via betting odds. However, there are several conclusions based upon such results.

Firstly, since no effects were found in return series on days after the match results, it can be said that using sporting events in investment strategies is not advised on Zagreb Stock Exchange. Even though the effects were not significant, it was often found that CAARs were negative on days after the match, regardless if the match was winning or losing. It seems that no profitable trading strategy could be formed on ZSE based upon football match results. Next, explanations of such results should be looked upon in future work. The results in this research are in line with psychological work such as Isen and Simmonds (1978), where the study obtained interesting results in gambling situations. Namely, positive results (such as winning a game in football match) caused a cautious optimism as authors defined it. This means that people with cautious optimism are more cautious and less likely to gamble; due to overestimating the probability of winning and underestimating the probability of losing. Other explanations could lie upon that majority of the investment portfolios of investors in Croatia have foreign assets compared to the domestic ones. In that way, the national pride and emotions which arise from any game do not reflect that much in the portfolios. This conclusion is in line with Botha and de Beer (2011), in which authors also did not find any significant effects of football outcomes due to great proportion of foreign investors on the stock market in South Africa. Similarly, Tufan (2004) concludes that results obtained in that research can be caused by the Istanbul Stock Exchange investor structure. As it is stated in the paper, almost half of Turkish 
shares are being held by foreigners. Also as a result, since the matches were held in different time zones, World Cup matches could not have any effect on ISE 100 Index returns. Finally, some explanations are given in Boyle and Walter (2003). Authors explained that if fans (which include investors as well) expect the team to win, no significant changes in the moods could be observed if the team actually wins. This has a consequence in results with a downward bias for positive CAARs. Similar can be stated for expecting the team to lose a game. To conclude, football clubs in Croatia are not stock companies as some other clubs in Europe are. Thus, this could add up to the explanations on no effects on stock returns as well.

\section{Conclusion}

In the recent decade a numerous number of studies which examine relationship between sporting events and stock market returns have been emerging. These studies argue that investors' mood changes with results from sporting events. As a consequence, this change is influencing investors' decision making process in portfolio management. One of these sporting events are football matches of the national team, where national pride comes in place and changes investor's mood depending on results from matches. In this context, this paper investigated whether stock market returns on Zagreb Stock Exchange are influenced by football match results of Croatia's national team between 2014 and 2018.

A conclusion is made that neither win, draw nor lose have an impact on stock price reaction on Zagreb Stock Exchange in analysed period. That is why no effective investment strategy regarding the observed stocks and the observed market could be recommended at this point. The study has some shortfalls. All of the stocks in the analysis were grouped into one sample. In that way, different sectors were grouped in all of the calculations (although previous literature does not separate the stocks in that way as well). Thus, further research can analyse the impact of football match results of Croatian national team on big tournaments such as European Championship or Fifa World Cup on different sectors in Croatia, such as tourism and beverages. However, the results in this research were found to be robust and this can be considered as initial attempt to get some insights into the considered topic. 


\begin{tabular}{|c|c|c|c|c|c|c|c|c|c|c|c|c|c|c|c|c|}
\hline & $E$ & $\underset{m}{\stackrel{N}{n}}$ & $\begin{array}{l}\mathscr{\infty} \\
\stackrel{\theta}{\varnothing}\end{array}$ & $\left|\begin{array}{c}\mathfrak{2} \\
\end{array}\right|$ & হे & $\frac{a}{0}$ & in & ஜ్ర & 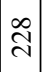 & $\stackrel{\infty}{\varrho}$ & $\frac{0}{a}$ & $\begin{array}{l}\infty \\
+ \\
q\end{array}$ & $\stackrel{ }{\varrho}$ & $\stackrel{\infty}{\stackrel{\infty}{\sim}}$ & 웅 & $\stackrel{\vartheta}{\exists}$ \\
\hline & के & $\begin{array}{l}\stackrel{0}{\circ} \\
\infty \\
\stackrel{0}{0} \\
\stackrel{0}{0}\end{array}$ & 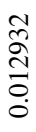 & $\mid \begin{array}{c}0 \\
\mathfrak{z} \\
\mathfrak{d} \\
0 \\
0 \\
0\end{array}$ & $\begin{array}{l}n \\
0 \\
0 \\
0 \\
0\end{array}$ & 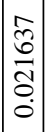 & $\begin{array}{l}0 \\
\infty \\
\infty \\
\tilde{\sigma} \\
0 \\
0\end{array}$ & 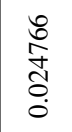 & 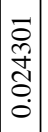 & $\begin{array}{l}\vec{\sigma} \\
\stackrel{0}{0} \\
\stackrel{0}{0}\end{array}$ & 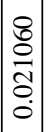 & $\begin{array}{l}\text { ro } \\
8 \\
0 \\
0\end{array}$ & 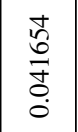 & 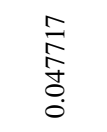 & $\begin{array}{l}\hat{n} \\
\stackrel{n}{\Omega} \\
0 \\
0\end{array}$ & \begin{tabular}{l}
8 \\
$\stackrel{0}{0}$ \\
\hdashline \\
0 \\
0
\end{tabular} \\
\hline & 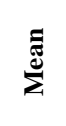 & 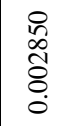 & 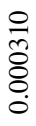 & 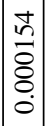 & 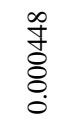 & 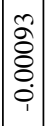 & 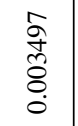 & 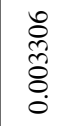 & 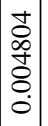 & $\begin{array}{l}\frac{n}{\pi} \\
\infty \\
8 \\
0\end{array}$ & 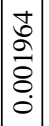 & 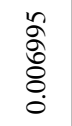 & $\begin{array}{l}8 \\
8 \\
8 \\
\dot{0}\end{array}$ & 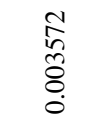 & $\begin{array}{l}\text { ơ } \\
\stackrel{1}{1} \\
8 \\
0\end{array}$ & $\begin{array}{l}\text { ले } \\
\tilde{\delta} \\
\stackrel{8}{0}\end{array}$ \\
\hline & 产 & 尝 & $\begin{array}{l}\text { 띠 } \\
\text { 은 }\end{array}$ & 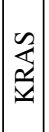 & 岂 & $\mid \begin{array}{l}\vec{\alpha} \\
\underline{d} \\
\mid\end{array}$ & $\underset{\vec{a}}{\vec{a}}$ & 㞼 & 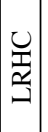 & 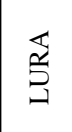 & $\frac{n}{\Sigma}$ & 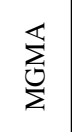 & 垉 & $\frac{a}{2}$ & $\underset{Z}{3}$ & 䓂 \\
\hline & 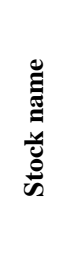 & 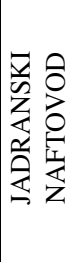 & 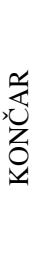 & 恣 & 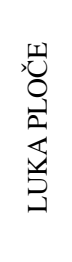 & 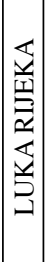 & 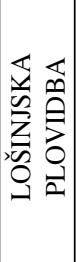 & 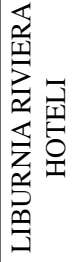 & 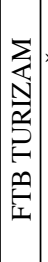 & 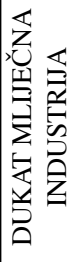 & 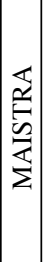 & 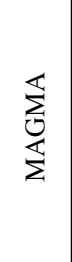 & 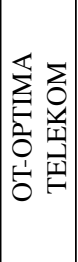 & 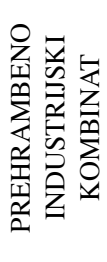 & 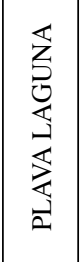 & $\begin{array}{l}\mathbb{8} \\
\frac{1}{2} \\
0\end{array}$ \\
\hline & & & & & & & & & & & & & & & & \\
\hline శ్ & $F$ & $\overrightarrow{\widehat{\omega}}$ & $\underset{\infty}{\infty}$ & 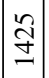 & $\begin{array}{l}\text { } \\
\stackrel{N}{\simeq}\end{array}$ & \begin{tabular}{l}
$\stackrel{8}{0}$ \\
\hdashline
\end{tabular} & 음 & $\underset{\Xi}{\Xi}$ & $\stackrel{?}{f}$ & $\vec{\infty}$ & $\underset{\mathrm{N}}{\mathrm{N}}$ & $\overrightarrow{\widetilde{N}}$ & $\stackrel{\widetilde{\mho}}{\Xi}$ & $\stackrel{\bigcirc}{\underset{\exists}{J}}$ & 导 & $\stackrel{\circ}{\circ}$ \\
\hline 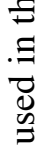 & के & $\begin{array}{l}\stackrel{N}{\sim} \\
\stackrel{n}{1} \\
0 \\
0\end{array}$ & 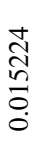 & 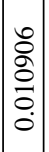 & $\begin{array}{l}n \\
n \\
\infty \\
0 \\
0\end{array}$ & 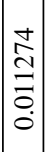 & $\begin{array}{l}\stackrel{8}{0} \\
\text { ते } \\
\text { ठ઼. }\end{array}$ & \begin{tabular}{l} 
Vo \\
\multirow{2}{n}{} \\
0 \\
$\stackrel{0}{0}$
\end{tabular} & 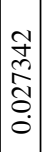 & 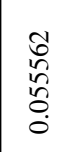 & $\mid \begin{array}{c}\hat{0} \\
0 \\
0 \\
0 \\
0 \\
0\end{array}$ & 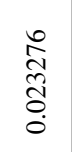 & $\begin{array}{l}\stackrel{\infty}{N} \\
\text { } \\
\text { రై } \\
\stackrel{0}{0}\end{array}$ & 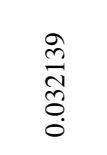 & 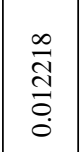 & 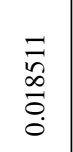 \\
\hline $\begin{array}{l}\frac{v}{8} \\
0 \\
\infty \\
8 \\
0 \\
\vdots\end{array}$ & $\sum^{\bar{\Xi}}$ & 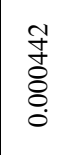 & $\begin{array}{l}+ \\
\infty \\
\infty \\
8 \\
8 \\
\stackrel{0}{0}\end{array}$ & 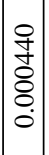 & $\begin{array}{l}\infty \\
\stackrel{0}{0} \\
\stackrel{0}{0} \\
\stackrel{0}{0}\end{array}$ & $\begin{array}{l}n \\
2 \\
2 \\
8 \\
8 \\
0\end{array}$ & 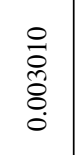 & 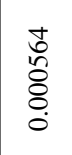 & 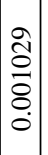 & $\begin{array}{l}\overrightarrow{8} \\
8 \\
8 \\
0\end{array}$ & 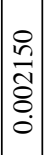 & 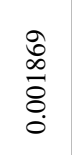 & $\begin{array}{l}\stackrel{\infty}{=} \\
\overline{8} \\
\stackrel{8}{0} \\
1\end{array}$ & $\begin{array}{l}\frac{1}{0} \\
\frac{0}{8} \\
0 \\
0\end{array}$ & 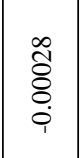 & 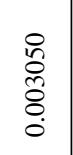 \\
\hline 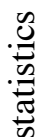 & : & $\overrightarrow{\hat{Z}}$ & $\frac{\tilde{U}}{2}$ & 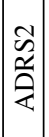 & 艺 & $\begin{array}{l}\text { 号 } \\
\text { 安 }\end{array}$ & 忌 & 完 & $\begin{array}{l}\text { 䍃 } \\
\text { 安 }\end{array}$ & ஸิ & $\underset{\sum}{\sum}$ & $\begin{array}{l}\tilde{0} \\
\text { ช̂ }\end{array}$ & $\stackrel{\overline{\vec{\imath}}}{\overrightarrow{0}}$ & $\overrightarrow{\vec{b}}$ & 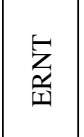 & 荘 \\
\hline 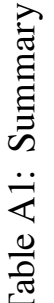 & 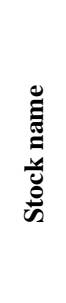 & 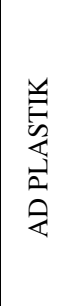 & 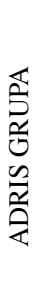 & 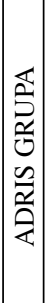 & 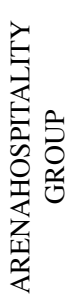 & 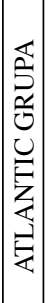 & 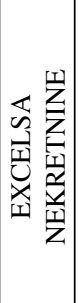 & 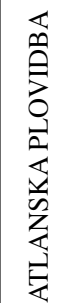 & 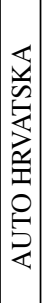 & 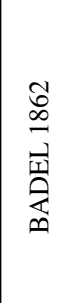 & 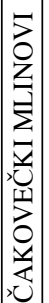 & 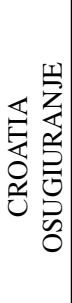 & 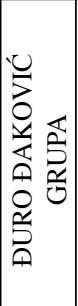 & $\begin{array}{l}0 \hat{0} \\
0 \\
0 \\
y \\
11 \\
\frac{1}{1}\end{array}$ & 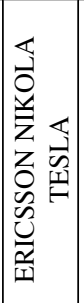 & 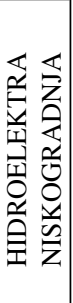 \\
\hline
\end{tabular}




\begin{tabular}{|c|c|c|c|c|c|c|c|c|c|c|c|c|c|c|c|}
\hline$E$ & లి & ప్రి & $\begin{array}{l}0 \\
0 \\
0\end{array}$ & กี & $\stackrel{\infty}{\leftrightarrow}$ & $\ni$ & 영 & $\begin{array}{l}n \\
n\end{array}$ & 2 & 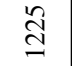 & $\overline{\tilde{\sigma}}$ & $\frac{0}{6}$ & ঙ̊ & $\begin{array}{l}\stackrel{0}{0} \\
\stackrel{I}{二}\end{array}$ & ષ્ત \\
\hline ซs & 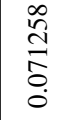 & $\begin{array}{l}\stackrel{n}{\rho} \\
\text { co } \\
0 \\
0\end{array}$ & $\mid \begin{array}{c}0 \\
n \\
\infty \\
2 \\
0 \\
0\end{array}$ & 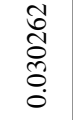 & $\begin{array}{l}\hat{\infty} \\
\infty \\
\infty \\
\tilde{0} \\
0\end{array}$ & 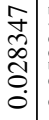 & $\begin{array}{l}n \\
\vdots \\
0 \\
\\
\vdots \\
0 \\
0\end{array}$ & 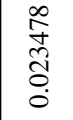 & 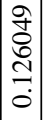 & 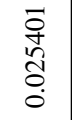 & $\left|\begin{array}{c}0 \\
\infty \\
o \\
O \\
0 \\
0 \\
0\end{array}\right|$ & $\begin{array}{l}\stackrel{2}{ } \\
\circ \\
\text { ते } \\
\stackrel{0}{0}\end{array}$ & $\begin{array}{l}\overrightarrow{0} \\
⿱ 0 \\
0 \\
0 \\
0\end{array}$ & $\begin{array}{l}\frac{d}{\sqrt{n}} \\
\frac{0}{0} \\
\stackrel{0}{0}\end{array}$ & $\begin{array}{l}\underset{D}{J} \\
\infty \\
⿱ \\
0 \\
0\end{array}$ \\
\hline$\underbrace{\tilde{\varpi}}$ & 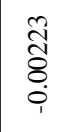 & $\begin{array}{l}\bar{\nabla} \\
\overline{8} \\
8 \\
0\end{array}$ & 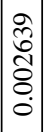 & $\begin{array}{l}\text { ते } \\
\widetilde{\delta} \\
0 \\
0\end{array}$ & $\begin{array}{l}\frac{n}{\infty} \\
\tilde{\delta} \\
0 \\
0\end{array}$ & $\begin{array}{l}a \\
\dot{\sigma} \\
\dot{8} \\
\dot{0}\end{array}$ & $\begin{array}{l}n \\
\vdots \\
\delta \\
8 \\
\vdots \\
1\end{array}$ & 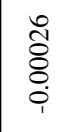 & 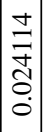 & 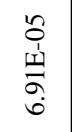 & $\mid \begin{array}{c}\infty \\
2 \\
2 \\
8 \\
0 \\
0\end{array}$ & $\begin{array}{l}\infty \\
\stackrel{\infty}{8} \\
\vdots \\
\vdots\end{array}$ & $\begin{array}{l}\overrightarrow{0} \\
8 \\
8 \\
8 \\
0\end{array}$ & 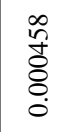 & $\begin{array}{l}\stackrel{2}{\hat{0}} \\
\text { oे } \\
8 \\
0 \\
0\end{array}$ \\
\hline لق. & $\sum$ & $\sum_{\simeq}^{\infty}$ & $\begin{array}{l}0 \\
\stackrel{y}{\simeq} \\
\mid\end{array}$ & 売 & $\begin{array}{l}\frac{\omega}{\omega} \\
\frac{1}{n}\end{array}$ & 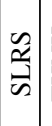 & $\begin{array}{l}\text { 光 } \\
\text { 壳 } \\
\end{array}$ & $\overbrace{Z}^{0}$ & $\mid \begin{array}{l}z \\
3 \\
5\end{array}$ & $\overrightarrow{\vec{b}}$ & $\mid \frac{\vec{z}}{5}$ & $\begin{array}{l}\stackrel{o}{\prime} \\
\stackrel{3}{>}\end{array}$ & 茎 & 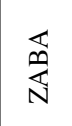 & $\stackrel{n}{N}$ \\
\hline 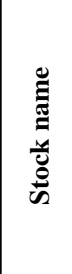 & 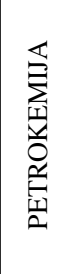 & 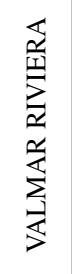 & 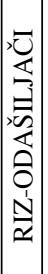 & 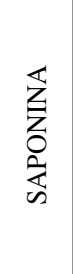 & 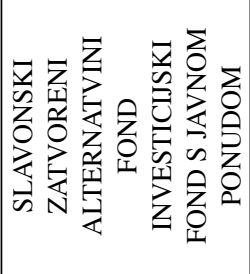 & 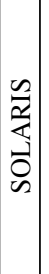 & 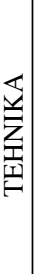 & 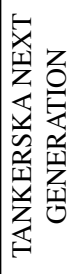 & 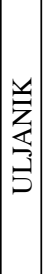 & 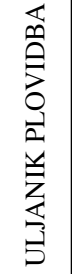 & 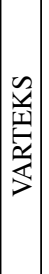 & 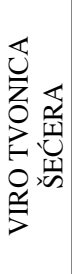 & 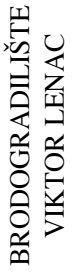 & 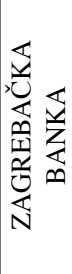 & 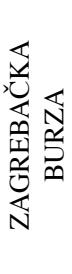 \\
\hline & & & & & & & & & & & & & & & \\
\hline$E$ & $\stackrel{\infty}{\sim}$ & f & $\frac{\infty}{6}$ & $\stackrel{\text { }}{\approx}$ & $\triangleright$ & $\begin{array}{l}n \\
\text { f } \\
\end{array}$ & $\stackrel{尺}{\stackrel{2}{*}}$ & $\stackrel{尺}{i}$ & $\frac{1}{\sigma}$ & $\widehat{\beth}$ & $\mathbb{I}$ & $\hat{\circ}$ & 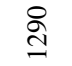 & 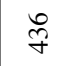 & ๙ૂ \\
\hline ซิ & 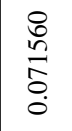 & 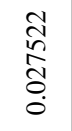 & $\mid \begin{array}{c}1 \\
\infty \\
\tilde{2} \\
\tilde{\sigma} \\
\dot{0}\end{array}$ & 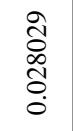 & 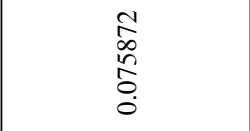 & $\begin{array}{l}n \\
m \\
\infty \\
0 \\
0 \\
0\end{array}$ & $\begin{array}{l}0 \\
0 \\
⿱ 亠 乂 \\
\vdots \\
0 \\
0 \\
0\end{array}$ & 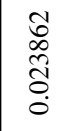 & 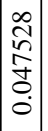 & $\begin{array}{l}\hat{\delta} \\
\tilde{0} \\
\tilde{\delta} \\
\stackrel{0}{0}\end{array}$ & 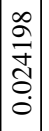 & $\begin{array}{l}\stackrel{8}{0} \\
\stackrel{+}{0} \\
o \\
0\end{array}$ & 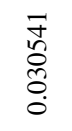 & 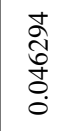 & 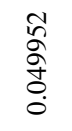 \\
\hline$\sum_{\Sigma}^{\bar{E}}$ & $\frac{\hat{\sigma}}{0}$ & $\begin{array}{l}\text { के } \\
\text { ते } \\
\text { ठ } \\
\stackrel{0}{0}\end{array}$ & 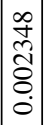 & 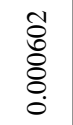 & $\begin{array}{l}\infty \\
\stackrel{\infty}{\hat{\sigma}} \\
\stackrel{8}{0}\end{array}$ & 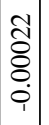 & 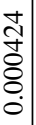 & 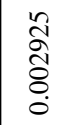 & $\mid \begin{array}{c}1 \\
\delta \\
\mathscr{\delta} \\
8 \\
0 \\
0\end{array}$ & 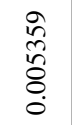 & $\mid \begin{array}{c}n \\
\hat{n} \\
6 \\
8 \\
0 \\
0\end{array}$ & $\begin{array}{l}\infty \\
\stackrel{\infty}{\infty} \\
\varnothing \\
\varnothing \\
0\end{array}$ & 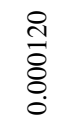 & \begin{tabular}{l}
$n$ \\
\multirow{2}{*}{} \\
$\tilde{\delta}$ \\
0 \\
0
\end{tabular} & 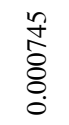 \\
\hline لق & 搨 & 兰 & 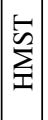 & 蜟 & 家 & $\underline{\Xi}$ & 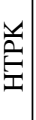 & $\stackrel{N}{\text { Ș }}$ & $\underset{\mathbb{J}}{\mathbb{D}}$ & $\begin{array}{l}\overleftrightarrow{n} \\
\Downarrow\end{array}$ & $\mid \begin{array}{l}\frac{\mathbb{\alpha}}{\exists} \\
=\end{array}$ & 艺 & 苍 & $\frac{1}{=}$ & $\overrightarrow{\hat{a}}$ \\
\hline 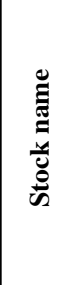 & 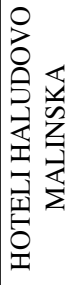 & 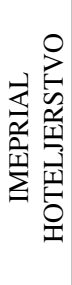 & 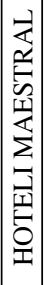 & 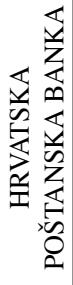 & 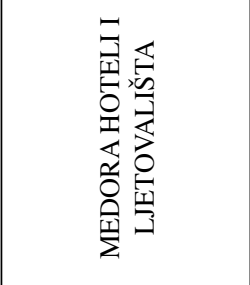 & 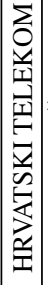 & 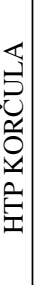 & 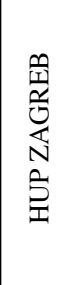 & 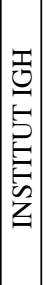 & 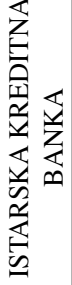 & 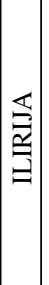 & 艺 & $\begin{array}{l}\text { 芯 } \\
\text { Z }\end{array}$ & 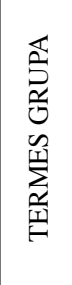 & 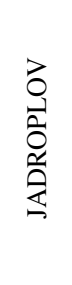 \\
\hline
\end{tabular}


Table A2: Event study results of football match results of Croatian national team on stock returns on Zagreb Stock Exchange, summary, expectations included

\begin{tabular}{|c|c|c|c|c|c|c|c|c|c|}
\hline \multirow{2}{*}{$\boldsymbol{t}$} & \multicolumn{3}{|c|}{ Win } & \multicolumn{3}{c|}{ Draw } & \multicolumn{3}{c|}{ Lose } \\
\cline { 2 - 10 } & $\mathbf{C A A R}_{\boldsymbol{t}}$ & $\boldsymbol{\theta}_{\mathbf{1}}$ & $\boldsymbol{\theta}_{\mathbf{3}}$ & $\mathbf{C A A R _ { t }}$ & $\boldsymbol{\theta}_{\mathbf{1}}$ & $\boldsymbol{\theta}_{\mathbf{3}}$ & CAAR $_{\boldsymbol{t}}$ & $\boldsymbol{\theta}_{\mathbf{1}}$ & $\boldsymbol{\theta}_{\mathbf{3}}$ \\
\hline \multirow{2}{*}{1} & 0.0009 & $\begin{array}{c}0.028 \\
(0.511)\end{array}$ & $\begin{array}{c}0.021 \\
(0.508)\end{array}$ & -0.0021 & $\begin{array}{c}-0.083 \\
(0.467)\end{array}$ & $\begin{array}{c}0.037 \\
(0.515)\end{array}$ & 0.0009 & $\begin{array}{c}0.039 \\
(0.515)\end{array}$ & $\begin{array}{c}0.035 \\
(0.514)\end{array}$ \\
\hline 0 & 0.0001 & $\begin{array}{c}0.001 \\
(0.501)\end{array}$ & $\begin{array}{c}0.021 \\
(0.509)\end{array}$ & 0.0007 & $\begin{array}{c}0.018 \\
(0.507)\end{array}$ & $\begin{array}{c}0.037 \\
(0.515)\end{array}$ & 0.0029 & $\begin{array}{c}0.071 \\
(0.528)\end{array}$ & $\begin{array}{c}0.034 \\
(0.514)\end{array}$ \\
\hline \multirow{2}{*}{1} & -0.0009 & $\begin{array}{c}-0.018 \\
(0.493)\end{array}$ & $\begin{array}{c}0.021 \\
(0.509)\end{array}$ & 0.0005 & $\begin{array}{c}0.009 \\
(0.503)\end{array}$ & $\begin{array}{c}0.039 \\
(0.516)\end{array}$ & 0.0033 & $\begin{array}{c}0.079 \\
(0.532)\end{array}$ & $\begin{array}{c}0.035 \\
(0.514)\end{array}$ \\
\hline \multirow{2}{*}{2} & 0.0017 & $\begin{array}{c}0.031 \\
(0.512)\end{array}$ & $\begin{array}{c}0.022 \\
(0.509)\end{array}$ & -0.0021 & $\begin{array}{c}-0.034 \\
(0.486)\end{array}$ & $\begin{array}{c}0.039 \\
(0.516)\end{array}$ & 0.0029 & $\begin{array}{c}0.056 \\
(0.522)\end{array}$ & $\begin{array}{c}0.034 \\
(0.514)\end{array}$ \\
\hline
\end{tabular}

Note: $p$-values are given in brackets.

Table A3: Event study results of football match results of Croatian national team on stock returns on Zagreb Stock Exchange, by each event for WIN, expectations included

\begin{tabular}{|c|c|c|c|c|c|c|}
\hline Date & $t$ & $\mathbf{C A A R}_{t}$ & $\boldsymbol{\theta}_{1}$ & p-v & $\boldsymbol{\theta}_{3}$ & p-v \\
\hline \multirow{4}{*}{31 May 2014} & -1 & -0.0100 & -0.353 & 0.362 & 0.083 & 0.533 \\
\hline & 0 & -0.0093 & -0.166 & 0.434 & 0.094 & 0.537 \\
\hline & 1 & 0.0047 & 0.070 & 0.528 & 0.125 & 0.550 \\
\hline & 2 & 0.0178 & 0.202 & 0.580 & 0.145 & 0.558 \\
\hline \multirow{4}{*}{6 June 2014} & -1 & 0.0121 & 0.340 & 0.633 & 0.128 & 0.551 \\
\hline & 0 & 0.0133 & 0.232 & 0.592 & 0.128 & 0.551 \\
\hline & 1 & 0.0078 & 0.101 & 0.540 & 0.086 & 0.534 \\
\hline & 2 & -0.0028 & -0.035 & 0.486 & 0.071 & 0.528 \\
\hline \multirow{4}{*}{18 June 2014} & -1 & 0.0007 & 0.031 & 0.512 & 0.104 & 0.541 \\
\hline & 0 & -0.0086 & -0.309 & 0.379 & 0.073 & 0.529 \\
\hline & 1 & -0.0137 & -0.326 & 0.372 & 0.062 & 0.525 \\
\hline & 2 & -0.0057 & -0.132 & 0.447 & 0.104 & 0.541 \\
\hline \multirow{4}{*}{4 September 2014} & -1 & 0.0020 & 0.103 & 0.541 & 0.089 & 0.536 \\
\hline & 0 & -0.0018 & -0.078 & 0.469 & 0.056 & 0.522 \\
\hline & 1 & 0.0084 & 0.236 & 0.593 & 0.089 & 0.536 \\
\hline & 2 & 0.0060 & 0.128 & 0.551 & 0.078 & 0.531 \\
\hline \multirow{4}{*}{9 September 2014} & -1 & 0.0009 & 0.039 & 0.516 & 0.083 & 0.533 \\
\hline & 0 & 0.0013 & 0.037 & 0.515 & 0.090 & 0.536 \\
\hline & 1 & 0.0078 & 0.162 & 0.564 & 0.115 & 0.546 \\
\hline & 2 & 0.0064 & 0.128 & 0.551 & 0.109 & 0.543 \\
\hline \multirow{4}{*}{10 October 2014} & -1 & -0.0063 & -0.308 & 0.379 & 0.091 & 0.536 \\
\hline & 0 & -0.0191 & -0.537 & 0.296 & 0.054 & 0.522 \\
\hline & 1 & -0.0366 & -0.704 & 0.241 & 0.054 & 0.522 \\
\hline & 2 & -0.0349 & -0.698 & 0.243 & 0.054 & 0.522 \\
\hline \multirow{4}{*}{13 October 2014} & -1 & -0.0128 & -0.461 & 0.322 & 0.082 & 0.533 \\
\hline & 0 & -0.0303 & -0.706 & 0.240 & 0.063 & 0.525 \\
\hline & 1 & -0.0286 & -0.683 & 0.247 & 0.045 & 0.518 \\
\hline & 2 & -0.0350 & -0.633 & 0.263 & 0.073 & 0.529 \\
\hline
\end{tabular}




\section{Table A3. Continued}

\begin{tabular}{|c|c|c|c|c|c|c|}
\hline Date & $t$ & CAAR $_{t}$ & $\boldsymbol{\theta}_{1}$ & p-v & $\boldsymbol{\theta}_{3}$ & p-v \\
\hline \multirow{4}{*}{28 March 2015} & -1 & -0.0018 & -0.102 & 0.459 & 0.120 & 0.548 \\
\hline & 0 & 0.0013 & 0.065 & 0.526 & 0.103 & 0.541 \\
\hline & 1 & 0.0056 & 0.134 & 0.553 & 0.103 & 0.541 \\
\hline & 2 & 0.0187 & 0.279 & 0.610 & 0.103 & 0.541 \\
\hline \multirow{4}{*}{7 June 2015} & -1 & 0.0023 & 0.116 & 0.546 & 0.086 & 0.534 \\
\hline & 0 & -0.0076 & -0.270 & 0.394 & 0.155 & 0.562 \\
\hline & 1 & -0.0298 & -0.407 & 0.342 & 0.120 & 0.548 \\
\hline & 2 & -0.0275 & -0.526 & 0.299 & 0.086 & 0.534 \\
\hline \multirow{4}{*}{10 October 2015} & -1 & 0.0047 & 0.091 & 0.536 & 0.063 & 0.525 \\
\hline & 0 & -0.0018 & -0.066 & 0.474 & 0.109 & 0.544 \\
\hline & 1 & -0.0056 & -0.174 & 0.431 & 0.109 & 0.544 \\
\hline & 2 & 0.0061 & 0.164 & 0.565 & 0.141 & 0.556 \\
\hline \multirow{4}{*}{13 October 2015} & -1 & -0.0043 & -0.228 & 0.410 & 0.125 & 0.550 \\
\hline & 0 & 0.0079 & 0.399 & 0.655 & 0.172 & 0.568 \\
\hline & 1 & -0.0084 & -0.222 & 0.412 & 0.156 & 0.562 \\
\hline & 2 & -0.0051 & -0.126 & 0.450 & 0.141 & 0.556 \\
\hline \multirow{4}{*}{17 November 2015} & -1 & -0.0054 & -0.318 & 0.375 & 0.128 & 0.551 \\
\hline & 0 & -0.0016 & -0.062 & 0.475 & 0.107 & 0.542 \\
\hline & 1 & 0.0013 & 0.046 & 0.518 & 0.171 & 0.568 \\
\hline & 2 & 0.0054 & 0.169 & 0.567 & 0.171 & 0.568 \\
\hline \multirow{4}{*}{23 March 2016} & -1 & 0.0093 & 0.777 & 0.781 & 0.210 & 0.583 \\
\hline & 0 & 0.0010 & 0.048 & 0.519 & 0.134 & 0.553 \\
\hline & 1 & 0.0006 & 0.034 & 0.514 & 0.134 & 0.553 \\
\hline & 2 & 0.0031 & 0.117 & 0.547 & 0.172 & 0.568 \\
\hline \multirow{4}{*}{27 May 2016} & -1 & -0.0013 & -0.066 & 0.474 & 0.115 & 0.546 \\
\hline & 0 & -0.0034 & -0.162 & 0.436 & 0.095 & 0.538 \\
\hline & 1 & 0.0018 & 0.093 & 0.537 & 0.115 & 0.546 \\
\hline & 2 & 0.0091 & 0.324 & 0.627 & 0.172 & 0.568 \\
\hline \multirow{4}{*}{4 June 2016} & -1 & -0.0005 & -0.015 & 0.494 & 0.177 & 0.570 \\
\hline & 0 & -0.0241 & -0.386 & 0.350 & 0.133 & 0.553 \\
\hline & 1 & -0.0272 & -0.599 & 0.274 & 0.133 & 0.553 \\
\hline & 2 & -0.0375 & -0.764 & 0.222 & 0.044 & 0.518 \\
\hline \multirow{4}{*}{12 June 2016} & -1 & 0.0086 & 0.835 & 0.798 & 0.270 & 0.606 \\
\hline & 0 & 0.0135 & 0.645 & 0.740 & 0.270 & 0.606 \\
\hline & 1 & 0.0131 & 0.873 & 0.809 & 0.324 & 0.627 \\
\hline & 2 & 0.0135 & 1.035 & 0.850 & 0.324 & 0.627 \\
\hline \multirow{4}{*}{21 June 2016} & -1 & -0.0031 & -0.403 & 0.344 & 0.082 & 0.533 \\
\hline & 0 & 0.0054 & 0.443 & 0.671 & 0.219 & 0.587 \\
\hline & 1 & 0.0008 & 0.080 & 0.532 & 0.164 & 0.565 \\
\hline & 2 & 0.0044 & 0.144 & 0.557 & 0.110 & 0.544 \\
\hline \multirow{4}{*}{6 October 2016} & -1 & 0.0007 & 0.035 & 0.514 & 0.103 & 0.541 \\
\hline & 0 & 0.0049 & 0.189 & 0.575 & 0.086 & 0.534 \\
\hline & 1 & 0.0028 & 0.094 & 0.537 & 0.086 & 0.534 \\
\hline & 2 & 0.0126 & 0.268 & 0.606 & 0.103 & 0.541 \\
\hline
\end{tabular}


Table A3. Continued

\begin{tabular}{|c|c|c|c|c|c|c|}
\hline Date & $t$ & CAAR $_{t}$ & $\theta_{1}$ & p-v & $\theta_{3}$ & $\mathbf{p}-\mathbf{v}$ \\
\hline \multirow{4}{*}{9 October 2016} & -1 & -0.0024 & -0.176 & 0.430 & 0.134 & 0.553 \\
\hline & 0 & 0.0082 & 0.190 & 0.575 & 0.134 & 0.553 \\
\hline & 1 & 0.0045 & 0.088 & 0.535 & 0.095 & 0.538 \\
\hline & 2 & 0.0076 & 0.133 & 0.553 & 0.115 & 0.546 \\
\hline \multirow{4}{*}{12 November 2016} & -1 & 0.0206 & 0.654 & 0.744 & 0.224 & 0.589 \\
\hline & 0 & 0.0261 & 0.690 & 0.755 & 0.224 & 0.589 \\
\hline & 1 & 0.0379 & 0.654 & 0.744 & 0.207 & 0.582 \\
\hline & 2 & 0.0448 & 0.525 & 0.700 & 0.189 & 0.575 \\
\hline \multirow{4}{*}{15 November 2016} & -1 & 0.0117 & 0.385 & 0.650 & 0.114 & 0.545 \\
\hline & 0 & 0.0167 & 0.265 & 0.605 & 0.086 & 0.534 \\
\hline & 1 & 0.0204 & 0.350 & 0.637 & 0.157 & 0.562 \\
\hline & 2 & 0.0165 & 0.307 & 0.621 & 0.128 & 0.551 \\
\hline \multirow{4}{*}{24 March 2017} & -1 & -0.0104 & -0.460 & 0.323 & 0.067 & 0.527 \\
\hline & 0 & -0.0112 & -0.440 & 0.330 & 0.078 & 0.531 \\
\hline & 1 & -0.0015 & -0.069 & 0.473 & 0.089 & 0.536 \\
\hline & 2 & 0.0115 & 0.344 & 0.635 & 0.101 & 0.540 \\
\hline \multirow{4}{*}{27 May 2017} & -1 & 0.0040 & 0.195 & 0.577 & 0.082 & 0.533 \\
\hline & 0 & 0.0040 & 0.266 & 0.605 & 0.164 & 0.565 \\
\hline & 1 & 0.0040 & 0.140 & 0.556 & 0.192 & 0.576 \\
\hline & 2 & 0.0076 & 0.273 & 0.607 & 0.164 & 0.565 \\
\hline \multirow{4}{*}{3 September 2017} & -1 & 0.0019 & 0.133 & 0.553 & 0.126 & 0.550 \\
\hline & 0 & -0.0055 & -0.380 & 0.352 & 0.063 & 0.525 \\
\hline & 1 & -0.0109 & -0.574 & 0.283 & 0.063 & 0.525 \\
\hline & 2 & $\begin{array}{l}-0.0088 \\
\end{array}$ & -0.424 & 0.336 & 0.063 & 0.525 \\
\hline \multirow{4}{*}{9 October 2017} & -1 & 0.0027 & 0.305 & 0.620 & 0.177 & 0.570 \\
\hline & 0 & 0.0006 & 0.053 & 0.521 & 0.177 & 0.570 \\
\hline & 1 & -0.0013 & -0.144 & 0.443 & 0.088 & 0.535 \\
\hline & 2 & -0.0002 & -0.014 & 0.495 & 0.177 & 0.570 \\
\hline \multirow{4}{*}{9 November 2017} & -1 & 0.0025 & 0.117 & 0.546 & 0.095 & 0.538 \\
\hline & 0 & 0.0065 & 0.168 & 0.567 & 0.153 & 0.561 \\
\hline & 1 & 0.0109 & 0.263 & 0.604 & 0.172 & 0.568 \\
\hline & 2 & 0.0097 & 0.185 & 0.573 & 0.172 & 0.568 \\
\hline \multirow{4}{*}{27 March 2018} & -1 & 0.0123 & 0.687 & 0.754 & 0.217 & 0.586 \\
\hline & 0 & 0.0039 & 0.212 & 0.584 & 0.168 & 0.567 \\
\hline & 1 & -0.0014 & -0.035 & 0.486 & 0.120 & 0.548 \\
\hline & 2 & 0.0012 & 0.031 & 0.513 & 0.144 & 0.557 \\
\hline \multirow{4}{*}{8 June 2018} & -1 & -0.0191 & -0.468 & 0.320 & 0.088 & 0.535 \\
\hline & 0 & -0.0146 & -0.420 & 0.337 & 0.221 & 0.587 \\
\hline & 1 & -0.0184 & -0.565 & 0.286 & 0.133 & 0.553 \\
\hline & 2 & -0.0200 & -0.519 & 0.302 & 0.133 & 0.553 \\
\hline \multirow{4}{*}{16 June 2018} & -1 & -0.0017 & -0.111 & 0.456 & 0.133 & 0.553 \\
\hline & 0 & -0.0035 & -0.252 & 0.400 & 0.088 & 0.535 \\
\hline & 1 & -0.0124 & -0.877 & 0.190 & 0.088 & 0.535 \\
\hline & 2 & -0.0094 & -0.602 & 0.273 & 0.088 & 0.535 \\
\hline
\end{tabular}


Table A3. Continued

\begin{tabular}{|c|c|c|c|c|c|c|}
\hline Date & $t$ & CAAR $_{t}$ & $\theta_{1}$ & p-v & $\boldsymbol{\theta}_{3}$ & $\mathbf{p}-\mathbf{v}$ \\
\hline \multirow{4}{*}{21 June 2018} & -1 & -0.0031 & -0.403 & 0.344 & 0.082 & 0.533 \\
\hline & 0 & 0.0054 & 0.443 & 0.671 & 0.219 & 0.587 \\
\hline & 1 & 0.0008 & 0.080 & 0.532 & 0.164 & 0.565 \\
\hline & 2 & 0.0044 & 0.144 & 0.557 & 0.110 & 0.544 \\
\hline \multirow{4}{*}{26 June 2018} & -1 & 0.0072 & 0.554 & 0.710 & 0.190 & 0.575 \\
\hline & 0 & 0.0031 & 0.376 & 0.646 & 0.253 & 0.600 \\
\hline & 1 & 0.0001 & 0.007 & 0.503 & 0.095 & 0.538 \\
\hline & 2 & -0.0066 & -0.273 & 0.392 & 0.063 & 0.525 \\
\hline \multirow{4}{*}{1 July 2018} & -1 & 0.0699 & 0.390 & 0.652 & 0.189 & 0.575 \\
\hline & 0 & 0.0692 & 0.390 & 0.652 & 0.189 & 0.575 \\
\hline & 1 & 0.0678 & 0.384 & 0.650 & 0.172 & 0.568 \\
\hline & 2 & 0.0726 & 0.405 & 0.657 & 0.172 & 0.568 \\
\hline \multirow{4}{*}{7 July 2018} & -1 & 0.0081 & 0.421 & 0.663 & 0.270 & 0.606 \\
\hline & 0 & 0.0019 & 0.197 & 0.578 & 0.270 & 0.606 \\
\hline & 1 & 0.0071 & 0.599 & 0.725 & 0.216 & 0.585 \\
\hline & 2 & 0.0069 & 0.387 & 0.651 & 0.216 & 0.585 \\
\hline \multirow{4}{*}{11 July 2018} & -1 & -0.0008 & -0.157 & 0.438 & 0.179 & 0.571 \\
\hline & 0 & -0.0013 & -0.170 & 0.432 & 0.268 & 0.606 \\
\hline & 1 & -0.0031 & -0.440 & 0.330 & 0.179 & 0.571 \\
\hline & 2 & -0.0030 & -0.509 & 0.305 & 0.089 & 0.536 \\
\hline \multirow{4}{*}{15 October 2018} & -1 & 0.0039 & 0.080 & 0.532 & 0.110 & 0.544 \\
\hline & 0 & 0.0201 & 0.190 & 0.575 & 0.082 & 0.533 \\
\hline & 1 & 0.0310 & 0.309 & 0.622 & 0.192 & 0.576 \\
\hline & 2 & 0.0383 & 0.373 & 0.645 & 0.164 & 0.565 \\
\hline \multirow{4}{*}{15 November 2018} & -1 & 0.0227 & 0.192 & 0.576 & 0.168 & 0.567 \\
\hline & 0 & 0.0346 & 0.290 & 0.614 & 0.168 & 0.567 \\
\hline & 1 & 0.0143 & 0.245 & 0.597 & 0.168 & 0.567 \\
\hline & 2 & 0.0118 & 0.138 & 0.555 & 0.168 & 0.567 \\
\hline
\end{tabular}

Table A4: Event study results of football match results of Croatian national team on stock returns on Zagreb Stock Exchange, by each event for DRAW, expectations included

\begin{tabular}{|c|c|c|c|c|c|c|}
\hline Date & $\boldsymbol{t}$ & $\mathbf{C A A R}_{\boldsymbol{t}}$ & $\boldsymbol{\theta}_{\mathbf{1}}$ & $\mathbf{p - v}$ & $\boldsymbol{\theta}_{\mathbf{3}}$ & $\mathbf{p - v}$ \\
\hline \multirow{4}{*}{ 5 March 2014 } & -1 & -0.016 & -0.637 & 0.262 & 0.078 & 0.531 \\
\cline { 2 - 7 } & 0 & -0.003 & -0.065 & 0.474 & 0.107 & 0.542 \\
\cline { 2 - 7 } & 1 & 0.004 & 0.077 & 0.531 & 0.107 & 0.542 \\
\cline { 2 - 7 } & 2 & 0.004 & 0.064 & 0.526 & 0.116 & 0.546 \\
\hline \multirow{3}{*}{ 16 November 2014 } & -1 & -0.002 & -0.150 & 0.440 & 0.079 & 0.531 \\
\cline { 2 - 7 } & 0 & -0.006 & -0.337 & 0.368 & 0.092 & 0.537 \\
\cline { 2 - 7 } & 1 & -0.006 & -0.303 & 0.381 & 0.092 & 0.537 \\
\cline { 2 - 7 } & 2 & -0.008 & -0.280 & 0.390 & 0.052 & 0.521 \\
\hline
\end{tabular}


Table A4. Continued

\begin{tabular}{|c|c|c|c|c|c|c|}
\hline Date & $t$ & $\mathbf{C A A R}_{t}$ & $\theta_{1}$ & p-v & $\boldsymbol{\theta}_{3}$ & p-v \\
\hline \multirow{4}{*}{12 June 2015} & -1 & 0.011 & 0.395 & 0.653 & 0.125 & 0.550 \\
\hline & 0 & 0.014 & 0.367 & 0.643 & 0.109 & 0.544 \\
\hline & 1 & 0.016 & 0.454 & 0.675 & 0.141 & 0.556 \\
\hline & 2 & 0.005 & 0.132 & 0.553 & 0.109 & 0.544 \\
\hline \multirow{4}{*}{3 September 2015} & -1 & 0.006 & 0.244 & 0.597 & 0.149 & 0.559 \\
\hline & 0 & 0.002 & 0.134 & 0.553 & 0.171 & 0.568 \\
\hline & 1 & 0.004 & 0.202 & 0.580 & 0.192 & 0.576 \\
\hline & 2 & -0.003 & -0.121 & 0.452 & 0.149 & 0.559 \\
\hline \multirow{4}{*}{26 March 2016} & -1 & -0.008 & -0.422 & 0.337 & 0.095 & 0.538 \\
\hline & 0 & -0.004 & -0.197 & 0.422 & 0.095 & 0.538 \\
\hline & 1 & -0.008 & -0.350 & 0.363 & 0.095 & 0.538 \\
\hline & 2 & -0.009 & -0.276 & 0.391 & 0.095 & 0.538 \\
\hline \multirow{4}{*}{17 June 2016} & -1 & -0.003 & -0.656 & 0.256 & 0.162 & 0.564 \\
\hline & 0 & -0.007 & -0.309 & 0.378 & 0.216 & 0.585 \\
\hline & 1 & -0.016 & -0.335 & 0.369 & 0.270 & 0.606 \\
\hline & 2 & 0.007 & 0.756 & 0.775 & 0.324 & 0.627 \\
\hline \multirow{4}{*}{5 September 2016} & -1 & -0.007 & -0.308 & 0.379 & 0.076 & 0.530 \\
\hline & 0 & -0.006 & -0.213 & 0.415 & 0.153 & 0.561 \\
\hline & 1 & -0.013 & -0.305 & 0.380 & 0.134 & 0.553 \\
\hline & 2 & -0.011 & -0.227 & 0.410 & 0.153 & 0.561 \\
\hline \multirow{4}{*}{6 October 2017} & -1 & 0.003 & 0.278 & 0.609 & 0.265 & 0.605 \\
\hline & 0 & 0.006 & 0.315 & 0.623 & 0.221 & 0.587 \\
\hline & 1 & 0.004 & 0.248 & 0.598 & 0.221 & 0.587 \\
\hline & 2 & 0.002 & 0.204 & 0.581 & 0.265 & 0.605 \\
\hline \multirow{4}{*}{12 November 2017} & -1 & -0.002 & -0.081 & 0.468 & 0.131 & 0.552 \\
\hline & 0 & -0.005 & -0.124 & 0.451 & 0.092 & 0.537 \\
\hline & 1 & -0.016 & -0.203 & 0.420 & 0.092 & 0.537 \\
\hline & 2 & -0.034 & -0.315 & 0.376 & 0.092 & 0.537 \\
\hline \multirow{4}{*}{6 September 2018} & -1 & 0.021 & 0.375 & 0.646 & 0.340 & 0.633 \\
\hline & 0 & 0.041 & 0.359 & 0.640 & 0.272 & 0.607 \\
\hline & 1 & 0.033 & 0.308 & 0.621 & 0.204 & 0.581 \\
\hline & 2 & 0.042 & 0.337 & 0.632 & 0.204 & 0.581 \\
\hline \multirow{4}{*}{12 October 2018} & -1 & -0.004 & -0.417 & 0.338 & 0.120 & 0.548 \\
\hline & 0 & 0.002 & 0.050 & 0.520 & 0.024 & 0.510 \\
\hline & 1 & 0.020 & 0.206 & 0.582 & 0.072 & 0.529 \\
\hline & 2 & 0.023 & 0.250 & 0.599 & 0.120 & 0.548 \\
\hline
\end{tabular}


Table A5: Event study results of football match results of Croatian national team on stock returns on Zagreb Stock Exchange, by each event for LOSE, expectations included

\begin{tabular}{|c|c|c|c|c|c|c|}
\hline Date & $t$ & CAAR $_{t}$ & $\theta_{1}$ & p-v & $\theta_{3}$ & p-v \\
\hline \multirow{4}{*}{12 June 2014} & -1 & -0.0001 & -0.003 & 0.499 & 0.131 & 0.552 \\
\hline & 0 & 0.0031 & 0.069 & 0.527 & 0.118 & 0.547 \\
\hline & 1 & 0.0038 & 0.072 & 0.529 & 0.079 & 0.531 \\
\hline & 2 & 0.0102 & 0.147 & 0.559 & 0.105 & 0.542 \\
\hline \multirow{4}{*}{23 June 2014} & -1 & -0.0042 & -0.207 & 0.418 & 0.105 & 0.542 \\
\hline & 0 & 0.0024 & 0.093 & 0.537 & 0.118 & 0.547 \\
\hline & 1 & 0.0030 & 0.092 & 0.537 & 0.157 & 0.562 \\
\hline & 2 & -0.0014 & -0.038 & 0.485 & 0.105 & 0.542 \\
\hline \multirow{4}{*}{12 November 2014} & -1 & -0.0028 & -0.124 & 0.451 & 0.097 & 0.539 \\
\hline & 0 & 0.0016 & 0.076 & 0.530 & 0.126 & 0.550 \\
\hline & 1 & 0.0017 & 0.059 & 0.523 & 0.116 & 0.546 \\
\hline & 2 & -0.0016 & -0.041 & 0.484 & 0.107 & 0.542 \\
\hline \multirow{4}{*}{6 September 2015} & -1 & -0.0050 & -0.374 & 0.354 & 0.096 & 0.538 \\
\hline & 0 & -0.0038 & -0.297 & 0.383 & 0.120 & 0.548 \\
\hline & 1 & -0.0100 & -0.545 & 0.293 & 0.096 & 0.538 \\
\hline & 2 & -0.0089 & -0.378 & 0.353 & 0.120 & 0.548 \\
\hline \multirow{4}{*}{25 June 2016} & -1 & -0.0090 & -0.348 & 0.364 & 0.148 & 0.559 \\
\hline & 0 & -0.0174 & -0.566 & 0.286 & 0.074 & 0.530 \\
\hline & 1 & -0.0145 & -0.440 & 0.330 & 0.111 & 0.544 \\
\hline & 2 & -0.0055 & -0.327 & 0.372 & 0.111 & 0.544 \\
\hline \multirow{4}{*}{28 March 2017} & -1 & 0.0147 & 0.749 & 0.773 & 0.145 & 0.558 \\
\hline & 0 & 0.0152 & 0.467 & 0.680 & 0.126 & 0.550 \\
\hline & 1 & 0.0149 & 0.305 & 0.620 & 0.097 & 0.539 \\
\hline & 2 & 0.0064 & 0.135 & 0.554 & 0.116 & 0.546 \\
\hline \multirow{4}{*}{11 June 2017} & -1 & 0.0037 & 0.161 & 0.564 & 0.155 & 0.562 \\
\hline & 0 & 0.0181 & 0.282 & 0.611 & 0.155 & 0.562 \\
\hline & 1 & 0.0287 & 0.432 & 0.667 & 0.172 & 0.568 \\
\hline & 2 & 0.0261 & 0.323 & 0.627 & 0.155 & 0.562 \\
\hline \multirow{4}{*}{5 September 2017} & -1 & -0.0028 & -0.177 & 0.430 & 0.110 & 0.544 \\
\hline & 0 & -0.0008 & -0.038 & 0.485 & 0.110 & 0.544 \\
\hline & 1 & -0.0026 & -0.109 & 0.457 & 0.137 & 0.555 \\
\hline & 2 & -0.0006 & -0.017 & 0.493 & 0.137 & 0.555 \\
\hline \multirow{4}{*}{23 March 2018} & -1 & -0.0040 & -0.332 & 0.370 & 0.076 & 0.530 \\
\hline & 0 & -0.0061 & -0.331 & 0.370 & 0.076 & 0.530 \\
\hline & 1 & 0.0030 & 0.136 & 0.554 & 0.095 & 0.538 \\
\hline & 2 & -0.0063 & -0.292 & 0.385 & 0.076 & 0.530 \\
\hline \multirow{4}{*}{3 June 2018} & -1 & -0.0037 & -0.411 & 0.340 & 0.110 & 0.544 \\
\hline & 0 & 0.0023 & 0.070 & 0.528 & 0.137 & 0.555 \\
\hline & 1 & 0.0038 & 0.093 & 0.537 & 0.164 & 0.565 \\
\hline & 2 & 0.0051 & 0.133 & 0.553 & 0.137 & 0.555 \\
\hline
\end{tabular}


Table A5. Continued

\begin{tabular}{|c|c|c|c|c|c|c|}
\hline Date & $\boldsymbol{t}$ & $\mathbf{C A A R}_{\boldsymbol{t}}$ & $\boldsymbol{\theta}_{\mathbf{1}}$ & $\mathbf{p}-\mathbf{v}$ & $\boldsymbol{\theta}_{\mathbf{3}}$ & $\mathbf{p}-\mathbf{v}$ \\
\hline \multirow{4}{*}{ 15 July 2018 } & -1 & -0.0017 & -0.190 & 0.425 & 0.268 & 0.606 \\
\cline { 2 - 7 } & 0 & -0.0016 & -0.282 & 0.389 & 0.179 & 0.571 \\
\cline { 2 - 7 } & 1 & -0.0020 & -0.287 & 0.387 & 0.268 & 0.606 \\
\cline { 2 - 7 } & 2 & 0.0004 & 0.031 & 0.512 & 0.179 & 0.571 \\
\hline \multirow{3}{*}{ 11 September 2018 } & -1 & 0.0038 & 0.164 & 0.565 & 0.221 & 0.587 \\
\cline { 2 - 7 } & 0 & 0.0264 & 0.250 & 0.599 & 0.133 & 0.553 \\
\cline { 2 - 7 } & 1 & 0.0148 & 0.240 & 0.595 & 0.221 & 0.587 \\
\cline { 2 - 7 } & 2 & 0.0393 & 0.391 & 0.652 & 0.265 & 0.605 \\
\hline \multirow{3}{*}{ 18 November 2018 } & -1 & 0.0134 & 0.368 & 0.644 & 0.137 & 0.555 \\
\cline { 2 - 7 } & 0 & -0.0076 & -0.112 & 0.455 & 0.164 & 0.565 \\
\cline { 2 - 7 } & 1 & -0.0101 & -0.190 & 0.425 & 0.137 & 0.555 \\
\cline { 2 - 7 } & 2 & -0.0141 & -0.169 & 0.433 & 0.164 & 0.565 \\
\hline
\end{tabular}

Note: $p$-values are given in brackets.

\section{NOTES}

${ }^{1}$ See Šego and Škrinjarić (2012) or Škrinjarić (2018b).

${ }^{2}$ See Škrinjarić (2018b) and references in this paper which refer to this market being interesting for exploiting inefficiencies.

${ }^{3}$ Authors also found that more important games, such as tournament matches, have bigger impact on share price movements, relative to less important games, such as friendly games.

${ }^{4} 2157$ matches played by 23 football teams in Europe from year 2007 until 2009.

${ }^{5}$ The test statistic is calculated from expression $\theta_{3}=\left(\frac{N^{+}}{N}-0.5\right) \frac{\sqrt{N}}{N}=\frac{N^{+}-N}{\sqrt{N}}$.

${ }^{6}$ Croatian stock market has problems with liquidity and the usual approach when using data from that market is to obtain the data on the most liquid stocks which basically make the majority of the stock market capitalization. See Minović (2012) or Vidović (2013) for further details. Moreover, Škrinjarić (2018a) states that in the period from September 2014 until May 2018, only 9 stocks on ZSE were traded at least $90 \%$ of the time, 17 stocks $75 \%, 25$ stocks $60 \%$ and only 37 stocks which were traded at least $30 \%$ of the time. Although there are a small number of stocks present which had great liquidity, we opted to use as much as data possible in the analysis due to parametric tests having greater degrees of freedom. For those stocks for which the data was missing around the event date, the algorithm excluded them from the analysis. Previous literature has shown that illiquidity is not an issue in mispricing on the stock market in event studies (see Tetlock, 2008). The algorithm of calculation is such that it uses all available data around the event. This means that some stocks were included for one game, and not for another. If we just used the most liquid ones, sometimes these stocks are not traded around event days. So, including as many stocks possible in the analysis, the data around each match was greater compared to starting with a smaller sample.

${ }^{7}$ The matches took place at the following dates: 6 February, 22 and 26 march, 7 and 10 June, 14 August, 6 and 10 September, 11 and 15 October and 15 and 19 November 2013.

${ }^{8}$ This effect was found in Šego and Škrinjarić (2012) on the Croatian market. Thus, we include it here as well. 
${ }^{9}$ Used stocks include all of the sectors represented on the ZSE; however, the sector classification is not necessary due to stocks not being divided into sectors in the analysis.

\section{REFERENCES}

Amélie, C. \& Olivier D. (2016). Stock market reactions to FIFA World Cup announcements: An event study. Economics Bulletin 36(4), 2028-2036.

Anand, C., Bahure, V., Balasubramaniam, V. \& Shah, A. (2017). Eventstudies R Package.

Ashton, J., Gerrard B. \& Hudson, R. (2003). Economic impact of national sporting success: evidence from the London stock exchange. Applied Economics Letters 10(12), 783-785. DOI: doi. org/10.1080/1350485032000126712

Baele, L., Bekaert, G. \& Schäfer, L. (2008). An anatomy of central and eastern European equity markets. European Bank for Reconstruction and Development, Working paper, No. 181.

Bartholdy, J., Olson, D. \& Peare, P. (2007). Conducting event studies on small stock exchange. The European Journal of Finance 13(3), 227-252. DOI: doi.org/10.1080/13518470600880176

Benkraiem, R., Louhichi, W. \& Marquès, P. (2009). Market reaction to sporting results: The case of European listed football clubs. Management Decision. 47: 100-109. DOI: 10.1108/00251740910929722.

Bentham, J. (1789). The principles of morals and legislation. New York: Macmillan (1948).

Berkowitz, J. P. \& Depken, C. A. (2018). A rational asymmetric reaction to news: evidence from English football clubs. Review of Quantitative Finance and Accounting, 51 (2), 347-374. DOI: doi.org/10.1007/s11156-017-0673-6.

Bernile, G. \& Lyandres, E. (2011). Understanding Investor Sentiment: The Case of Soccer. Financial Management 40(2), 357-380. DOI: doi.org/10.2139/ssrn.1343685

Berument, H. \& Ceylan, N. B. (2012). Effects of soccer on stock markets: The return-volatility relationship. The Social Science Journal 49(3), 368-374. DOI: doi.org/10.1016/j.soscij.(2012).03.003

Bloomfield, R., O'Hara, M. \& Saar, G. (2009). How Noise Trading Affects Markets: An Experimental Analysis. The Review of Financial Studies 22(6), 2275-2302. DOI: doi.org/10.1093/rfs/ hhn102

Botha, F. \& de Beer, C. (2011). Does National Sporting Performance affect Stock Market Returns in South Africa?. Journal of Economic and Financial Sciences 6(1), 67-82.

Boyle, G. \& Walter, B. (2003). Reflected glory and failure: international sporting success and the stock market. Applied Financial Economics 13(3), 225-235. DOI: doi. org/10.1080/09603100210148230

Brown, S. (1993). Fundamentals or Noise? Evidence from the Professional Basketball Betting Market. The Journal of Finance 48(4), 1193-1209. DOI: doi.org/10.2307/2329035

Castellani, M., Pieropaolo, P. \& Patuelli, R. (2012). Event Clustering and Abnormal Returns: Reassessing the Informational Value of Bets. Quaderni DSE working paper No. 817.

Corrado, C. (1989). A non parametric test for abnormal security price performance in event studies. Journal of Financial Economics 23, 385-395. DOI: doi.org/10.1016/0304-405X(89)90064-0

Croatian Football Federation (2018). Statistics, available at http://hns-cff.hr/info/statistika/

Demirhan, D. (2013). Stock Market Reaction to National Sporting Success: Case of Borsa Istanbul. Pamukkale Journal of Sport Sciences 4(3), 107-121.

Edmans, A., García, D. \& Norli, Ø. (2007). Sports Sentiment and Stock Returns, The Journal of Finance 62(4), 1967-1998. DOI: doi.org/10.1111/j.1540-6261.(2007).01262.x

Fama, E. (1965). The Behavior of Stock-Market Prices. Journal of Business 38(1), 34-105. 
Fama, E. (1970). Efficient Capital Markets: A Review of Theory and Empirical Work. The Journal of Finance 25(2), 383-417.

Floros, C. (2014). Football and Stock Returns: New Evidence. Procedia Economics and Finance 14(2014), 201- 209.

Gallagher, R \& O'Sullivan, N. (2011). Asset Pricing arising from Sports Results and investor Mood: The Case of a Homogenous Fan Base Area. Applied Financial Economics 21(12), 863-880.

Gerlach, J. R. (2011). International Sports and Investor Sentiment: Do National Team Matches Really Affect Stock Market Returns?. Applied Financial Economics 21(12), 863-880. DOI: doi. org/10.1080/09603107.(2010).543069

Hirshleifer, D. (2001). Investor Psychology and Asset Pricing. Journal of Finance 56(4), 1533-1597. DOI: doi.org/10.1111/0022-1082.00379

Isen, A. M. \& Simmonds, S. (1978). The Effect of Feeling Good on a Helping Task That Is Incompatible with Good Mood. Social Psychology 41(4), 346-349.

Isen, A. M., Nygren, T. \& Ashby, G. (1988). Influence of Positive Affect On The Subjective Utility Of Gains And Losses: It Is Just Not Worth The Risk. Journal of Personality and Social Psychology 55(5), 710-17.

Johnson, E \& Tversky. A. (1983). Affect, generalization, and the perception of risk. Journal of personality and social psychology 45(1), 20-31. DOI: doi.org/10.1037/0022-3514.45.1.20

Klein, C., Zwergel, B. \& Heiden, S. (2009). On the Existence of Sports Sentiment: The Relation between Football Match Results and Stock Index Returns in Europe. Review of Managerial Science 3(3), 191-208. DOI: doi.org/ 10.1007/s11846-009-0031-8

Lalić, D. (2018). Football and politics - History and sovereignty of interrelation in Croatia (In Croatian). Zaprešić: Fraktura d.o.o.

Levy, N. (2015). The Effect of New York City Sports Outcomes on the Stock Market. Undergraduate Economic Review 12(1), Article 8.

Linnainmaa, J. (2007). The Limit Order Effect. The Anderson School at UCLA, Working paper.

Loewenstein, G. 2000. Emotions in Economic Theory and Economic Behavior. Preferences, Behavior and Welfare 90(2), 427-432

Loewenstein, G., Weber, E., Hsee, C. \& Welch, N. (2001). Risk as feelings. Psychological bulletin 127(2), 267-286. DOI: doi.org/10.1037/0033-2909.127.2.267

MacKinlay, C. (1997). Event Studies in Economics and Finance. Journal of Economic Literatura 35(1), $13-39$.

Minović, J. (2012). Liquidity of the Croatian Stock Market: An Empirical Analysis. Economic Research - Ekonomska istraživanja 25(3), 776-801.

Newey, W. \& West, K. (1987). A Simple, Positive Semi-definite, Heteroskedasticity and Autocorrelation Consistent Covariance Matrix. Econometrica, 55(3),703-708. DOI: doi.org/10.2307/1913610.

Oddsportal (2019) http://www.oddsportal.com [accessed 15 February (2019)]

Palomino, F. A., Renneboog, L. D. R., \& Zhang, C. (2005). Stock Price Reactions to Short-Lived Public Information: The Case of Betting Odds. (CentER Discussion Paper; Vol. 2005-62).

Scholtens, B. (2009). Scoring on the stock exchange? The effect of football matches on stock market returns: an event study. Applied Economics 41(25), 3231-3237. DOI: doi. org/10.1080/00036840701721406

Šego, B. \& Škrinjarić, T. (2012). Modeliranje dnevne sezonalnosti prinosa na ZSE (Modelling daily seasonality in stock returns on ZSE), Aljinović, Z., Marasović, B. (ed.):: Matematički modeli u analizi razvoja hrvatskog financijskog tržišta (Mathematical models in analysis of the development of the Croatian financial market). University of Split: Faculty of Economics: 159-172.

Serra, A. P. (2002). Event Study Tests. A Brief Survey. Working papers Da Fep, Investigação - Trabalhos em curso - $\mathrm{n}^{\circ}$ 117, Maio de 2002. 
Sheskin, D. (2004). Handbook of Parametric and Nonparametric Statistical Procedures. CRC Press.

Škrinjarić, T. (2018a). Revisiting Herding Investment Behavior on the Zagreb Stock Exchange: A Quantile Regression Approach. Econometric Research in Finance 3(1), 119-162.

Škrinjarić, T. (2018b). Testing for seasonal affective disorder on selected CEE and SEE markets. Risks 6 (5), 1-26. DOI: doi.org/10.3390/risks6040140

Tetlock, P. C. (2008). Liquidity and Prediction Market Efficiency. SSRN papers. DOI: doi.org/10.2139/ ssrn.929916

Tetlock, P. C. (2011). All the News That's Fit to Reprint: Do Investors React to Stale Information?. The Review of Financial Studies 24(5), 1481-1512. DOI: doi.org/10.1093/rfs/hhq141

Toraman, C., Seyhan, M., Buğan, M. F. \& Yunus, K. (2016). The Relationship between National Football Teams' Performance and Stock Markets. International Journal of Academic Research in Business and Social Sciences. 6. 588-598. DOI: doi.org/10.6007/IJARBSS/v6-i12/2520.

Tufan, E. (2004) Do World Cup Football Matches Affect Istanbul Stock Exchange?. SSRN Electronic Journal. DOI: doi.org/10.2139/ssrn.705343.

Vidović, J. (2013). Investigation of stock illiquidity on Central and South East European markets in naïve portfolio framework. Ekonomska misao i praksa (2013)(2), 537-550.

Wann, D., Dolan, T., Mcgeorge, K. \& Allison, J. (1994). Relationships between spectator identification and spectators' perceptions of influence, spectators' emotions, and competition outcome. Journal of Sport and Exercise Psychology 16(4), 347-364. DOI: doi.org/10.1123/jsep.16.4.347

White, H. (1980). A heteroskedasticity-consistent covariance matrix estimator and direct test for heteroskedasticity. Econometrica 48(4), 285-316. doi.org/10.2307/1912934.

Zagreb Stock Exchange. (2018). http://www.zse.hr [accessed 5 December (2018)]

Zawadziki, K. (2013). The impact of mega sports events on the stock markets. MPRA Paper No. 44467

Zuber, R. A., Yiu, P., Lamb, R. \& Gandar, J. (2005). Investor-fans? An Examination of the Performance of Publicly Traded English Premier League Teams. Applied Financial Economics 15 (5), 305-313. DOI: doi.org/10.1080/0960310042000338713 